\title{
Las "cartas de los caciques" de Yucatán de 1567: nuevas perspectivas. Aportaciones desde la edición crítica y la traducción ${ }^{1}$
}

\author{
The "Letters from the Caciques" of Yucatan of 1567: \\ New Perspectives. Contributions from the Critical Edition \\ and Translation
}

\author{
Zoraida Raimúndez ARes \\ Posgrado en Arqueología e Historia, Universidad Complutense de Madrid \\ Posgrado en Estudios Mesoamericanos, Universidad Nacional Autónoma de México
}

\begin{abstract}
Resumen: Las "cartas de los caciques" son una serie de peticiones escritas en maya yucateco y en castellano. Utilizadas como fuente para la historia de Yucatán, éstas han sido estudiadas desde los textos castellanos, obviando el maya. Este artículo pone de manifiesto la importancia de conocer y trabajar con los documentos escritos en lenguas indígenas. Un análisis en profundidad de estas cartas revela que su elaboración se realizó de una forma particular, traducidas de los textos en castellano y no al contrario como se había supuesto hasta el momento. Esto es evidente si se compara con otros documentos del momento como la aquí denominada "Carta 8".

El análisis de la versión de las cartas escritas en maya, permite observar que contienen traducciones literales del texto en castellano y expresiones poco habituales, en contraste con otros documentos originalmente elaborados por los indígenas en sus propias lenguas. La génesis del documento aclaran y explican las razones de creación de estas cartas.
\end{abstract}

Palabras clave: Traducción, caciques, cartas coloniales, lengua maya, Yucatán.

AвSTRACT: The "Letters of the caciques" are a group of petitions written in Yucatec Maya and in Spanish. Used as a source of the Yucatan history, they have been studied from the Spanish version, obviating the one in Yucatec Maya. This article argues the importance of knowing and working with the indigenous texts. A deep analysis of these letters shows that they were elaborated in a very particular way, translated from a Spanish text and not the opposite as it had been assumed until

${ }^{1}$ En homenaje al doctor Alfonso Lacadena García-Gallo, ya que sin su apoyo y dirección no hubiera sido posible la realización de este trabajo. Mis agradecimientos al doctor José Alejos García por sus comentarios y su impulso para realizar esta publicación. El presente artículo fue presentado en el $X$ Congreso Internacional de Mayistas (2016), donde obtuvo el 2 o lugar en el "Premio Alberto Ruz Lhuillier". 
the present. This becomes obvious if these letters are compared with other similar documents like the one I call Letter 8.

The analysis of the Maya versions show a word by word translation from the Spanish text, and very unusual expressions, if contrasted with other original documents written by indigenous people in their own languages. The context and genesis of these documents explain the reasons and the surrounding circumstances involving the creation of this letters.

KeYwords: Translation, caciques, Colonial letters, Maya language, Yucatán.

RECEPCIÓN: 23 de octubre de 2018.

ACEPTACIÓN: 5 de diciembre de 2018.

DOI: 10.19130/iifl.ecm.2019.54.979

\section{Introducción}

Los documentos sobre los que se ha realizado la presente investigación consisten en un grupo de siete cartas escritas en maya yucateco y en castellano que integran un mismo corpus documental, presentando una serie de características comunes que las unifican tanto por su contenido y su aspecto similar, como por las fechas en que fueron realizadas.

La primera evidencia de este conjunto de documentos aparece en Cartas de Indias, una edición del Ministerio de Fomento de España realizada en 1877, en la que se presenta el documento que aquí se ha denominado Carta 1 (Cartas de Indias, 1877: 367-368) $)^{2}$ y que actualmente se ubica en el Archivo Histórico Nacional $^{3}$ en Madrid. ${ }^{4}$ Posteriormente, Günter Zimmerman presenta una recopilación de éste y otros documentos relativos al reinado de Felipe II en su obra Briefe der Indianischen Nobilität aus Neuspanien an Karl V und Philipp II um die Mitte des 16, en la que ofrece un total de seis "cartas de los caciques" de Yucatán (Zimmerman, 1970: 32-35), cinco de las cuales se encuentran en el Archivo General de Indias ${ }^{5}$ de Sevilla ${ }^{6}$ junto con el mencionado documento del AHN. En su obra ofrece una transcripción de los documentos, apuntando las diferencias existentes entre ellos y organizando las cartas por las provincias a las que se asocian los caciques firmantes del momento, junto con los facsímiles de las Cartas 4 y 6 (Zimmerman, 1970: 30-31). A partir de Zimmerman, varios investigadores han utilizado estos

${ }^{2}$ El facsímil de este documento se encuentra en el Anexo de la citada obra, denominado Facsímil U.

${ }^{3}$ En adelante AHN.

${ }^{4}$ AHN, Diversos-colecciones, 24, N. 77, 1585.

${ }^{5}$ En adelante AGI.

${ }^{6}$ Clasificación AGI, México, 367, ff. 62r-71v. El orden para la denominación de los documentos se corresponde con el de las fechas en las que fueron redactados. En los casos en los que éstas son coincidentes se ha seguido el orden que presentan en el AGI. Las referencias específicas para cada uno de los archivos son: Carta 2 (AGI, México, 367, ff. 70r-71v), Carta 3 (AGI, México, 367, ff. 62r-63v), Carta 4 (AGI, México, 367, ff. 64r-65v), Carta 5 (AGI, México, 367, ff. 66r-67v) y Carta 6 (AGI, México, 367 , ff. 68r-69v). 
documentos como fuente para el estudio y la reconstrucción de la historia de los primeros años de la Colonia. ${ }^{7}$

Hasta el momento, se ha considerado que tales documentos fueron escritos por los caciques en su lengua y que, posteriormente, fueron traducidos. Sin embargo, esta consideración es una simplificación del alcance y contenido de los escritos. En efecto, una traducción crítica y el estudio del contexto del momento revelan que el proceso de creación de estos documentos se realiza a partir de un original en castellano del que se traducen los documentos al maya en las diferentes localidades que señalan las cartas.

\section{Una nueva carta de los caciques de Yucatán a Felipe II}

Durante la investigación realizada (Raimúndez, 2015) sobre las "cartas de los caciques" de 1567, apareció en el AGI una nueva carta. El nuevo documento, que se ha denominado Carta $7,{ }^{8}$ es una carta inédita que aparece en el mismo legajo que el resto de cartas que se encuentran en Sevilla, pero separada del resto de documentos. Sus características son similares, con la salvedad de que ésta data del 5 de marzo de 1567 y las otras son del 11 y 12 de febrero del mismo año. Dicho documento aumenta el listado presentado por Zimmerman (1970: 32-35) a siete cartas, ampliando la zona de realización de las cartas hacia el sur de la península de Yucatán (Mapa 1). La ausencia de documentos procedentes de otras zonas con cabeceras importantes sugiere la posibilidad de que existan más cartas y que de esta forma aumente el corpus, haciéndolo mucho más complejo y extenso de lo que es en la actualidad.

Aquí se presenta, en los Anexos 1, 2 y 3, la Carta 7 que incluye el facsímil y su transliteración así como la traducción con el análisis, respectivamente.

\section{Metodología para el estudio del corpus}

La presente investigación propone como objetivo principal el estudio de documentos originales en lengua maya de época colonial a partir de las premisas de la "nueva filología" (Lockhart, 1992; Restall, 2001) en conjunto con la edición crítica de textos (Raimúndez, 2015). Tal metodología permite comprender aspectos no estudiados con anterioridad en los documentos coloniales en lenguas indígenas, en este caso el maya yucateco, y entender la génesis de este corpus.

\footnotetext{
${ }^{7}$ Entre los numerosos autores que han publicado acerca de estos documentos podemos destacar, por ejemplo, González Cicero (1978), Farris (1991), Quezada (1993, 2014), Restall (1997), Bracamonte y Sosa (2001), Cledinnen (2003), Chuchiak (2005), Hanks (2010) y Cunill (2012).

${ }^{8}$ AGI, México, 367, ff. 88r-89v.
} 


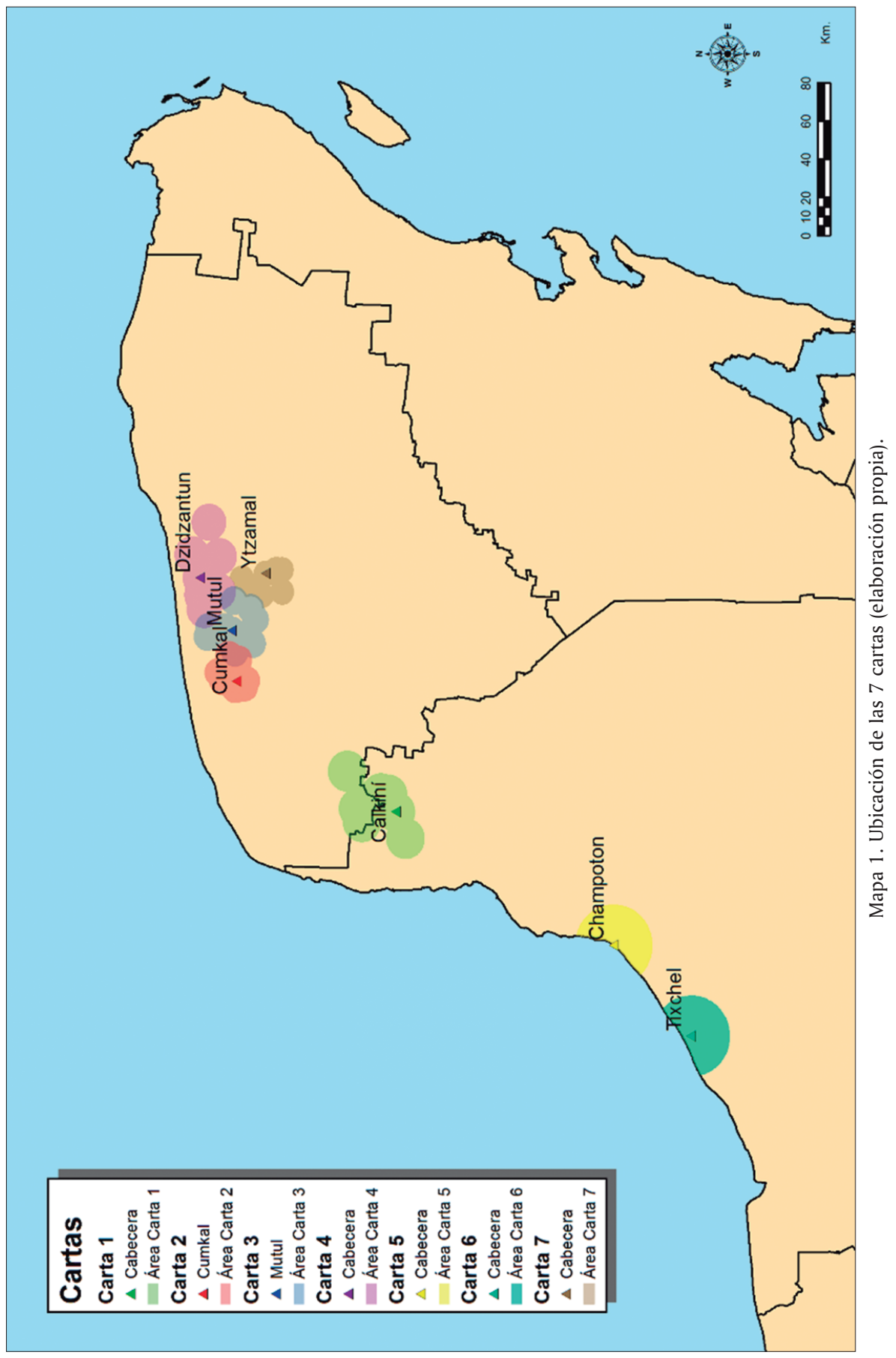


Los trabajos dedicados a la documentación colonial, tradicionalmente no han tomado en cuenta los documentos originales, ya sea utilizando la traducción realizada en la propia época, presentándose como textos bilingües, o empleando las traducciones elaboradas por especialistas mucho tiempo después. Esto ha supuesto un problema de pérdida de información, principalmente por la falta de una metodología crítica rigurosa a la hora de editar los textos. La finalidad del estudio ha sido obtener información de las cartas adoptando otra perspectiva, que sería el trabajo sobre el texto escrito en maya, contrastando la información obtenida de la traducción propia con el documento en castellano del original.

Los resultados de la aplicación de esta metodología sugieren que las cartas no fueron creadas por los propios caciques, sino que circuló un modelo escrito en castellano, que fue la base para la traducción de los textos mayas de dichas cartas. La idea de que los documentos no fueron iniciativa de los propios caciques, sino de otros interesados en su elaboración, es decir, los frailes franciscanos, ha sido sugerida por algunos investigadores como Stella María González Cicero (1978: 110-111) o Inga Clendinnen (2003: 101). Ambas sostienen que la espontaneidad de la creación de estos documentos es poco probable debido a su similitud y cercanía en fechas, siendo los franciscanos los que tenían los medios para manejar y obligar a los indígenas para que se unieran a sus peticiones.

Las premisas de la "nueva filología" desarrolladas por James Lockhart se aplican principalmente al náhuatl y, en este caso, se extrapolan al estudio de la documentación maya, partiendo de la idea de la importancia de los documentos escritos en lenguas indígenas que complementan los registros en castellano, proporcionando una mayor riqueza y comprensión de estos (Lockhart, 1992: 18) y favoreciendo a su vez el estudio etnohistórico (Rojas, 2008). Junto con las ideas de Lockhart, aquí se plantea un método basado en la edición crítica por el cual se combinan diversas maneras de abordar el estudio del documento. Se utilizan tanto la paleografía y diplomática tradicional aplicadas a los documentos escritos en español como el "método codicológico" (Batalla, 2008) y los aportes de la metodología empleada en la epigrafía maya. ${ }^{9}$ La utilización de las disciplinas mencionadas se basa en un estudio de los aspectos externos e internos del documento, la cual consiste en efectuar, en primer lugar, un análisis del soporte, estableciendo la tipología, autenticidad, estado de conservación y estudio de las filigranas ${ }^{10}$ (Figura 1) si se trata de papel europeo y, en segundo, un estudio de la grafía para determinar los escribanos participantes. Dentro de los aspectos internos se establecen las partes del documento desde la perspectiva de la diplomática y se acompaña de una traducción en la que se aplican y explicitan cinco pasos partiendo del documento original (Anexo 1).

\footnotetext{
${ }^{9}$ Este método fue planteado por el doctor Alfonso Lacadena y el Seminario Permanente de "Paleografía y gramática del maya yucateco colonial” del Departamento de Historia de América II (Antropología de América) de la Universidad Complutense de Madrid (2014-2016).

${ }^{10}$ La filigrana perteneciente a la Carta 7 permite corroborar la autenticidad del documento ya que lo asocia a una época específica que coincide con la fecha de su elaboración.
} 


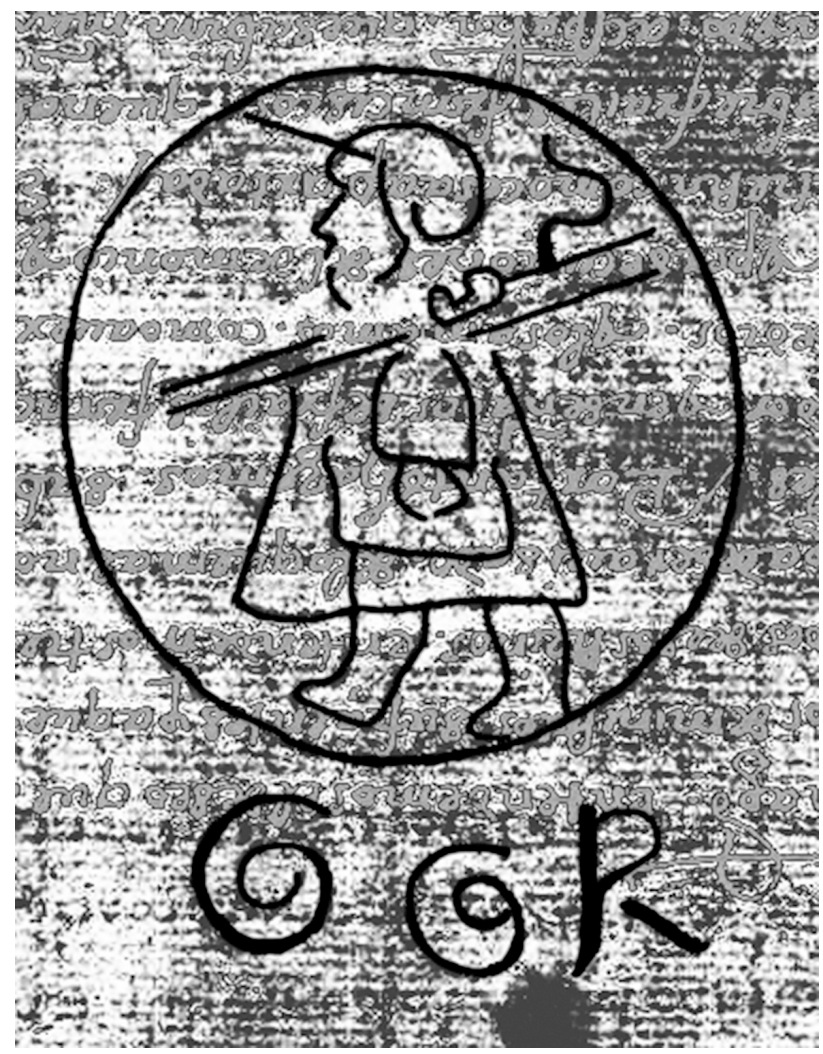

Figura 1. Dibujo de filigrana Carta 7 (elaboración propia).

Estos cinco pasos, propuestos por Alfonso Lacadena, permiten mostrar el proceso completo de traducción estableciendo una base firme, a partir de la cual se puede trabajar la información aportada por el texto y las posteriores interpretaciones que derivan de él. Como primer paso, se realiza la transliteración, por la cual se transcribe el texto arreglando la unión de las palabras correctamente, se mantienen todos los elementos del documento, se numeran las líneas acorde con el original y se utilizan símbolos especiales en la medida de lo posible, intentando respetar el original del documento; el segundo paso es la transcripción del documento, donde se corrigen las mayúsculas y minúsculas ortográficas, añadiendo los signos de puntuación necesarios y se desarrollan las abreviaturas; el tercer paso es la segmentación morfológica, en la que se identifican las partes constituyentes de las palabras; en un cuarto paso se analiza morfológicamente el texto, otorgando las funciones y categorías a cada una de las partes de las palabras separadas en el paso previo; $y$, finalmente, el quinto paso consiste en la traducción del texto. A pesar de que esta metodología se explica con pasos ordenados consecutivos, la traducción es un proceso complejo, ya que 
simultáneamente se deben realizar interpretaciones de las ideas expresadas por otra cultura, toma de decisiones para realizar una traducción comprensible y rectificaciones que llevan al texto finalmente mostrado.

\section{Contexto de las "cartas de los caciques"11}

Los antecedentes de gestación de las cartas se encuentran en 1562, en los acontecimientos que tuvieron lugar durante y tras el famoso Auto de Fe de Maní. La importancia de la participación en estos hechos de fray Diego de Landa se relaciona directamente con el corpus documental. En el contenido del documento se hace mención directa a su persona y a otros dos frailes, fray Miguel de la Puebla y fray Pedro Gumiel (Anexo 2, líneas 14, 15 y 18). Por otro lado, los juicios derivados de este Auto de Fe, que tuvieron lugar tanto en la península de Yucatán como en España, tienen también su consideración dentro de la génesis y contenido de los documentos.

Fray Diego de Landa había llegado a la península de Yucatán en 1549, llevado por fray Nicolás de Albalate, junto con un grupo de frailes. Landa, como franciscano conocedor de la lengua maya, comienza a desempeñar su labor misionera en el noroeste de la Península de Yucatán (Cogolludo, 1955, II: 73). En 1561 es nombrado ministro provincial de Yucatán (Scholes y Adams, 1938, I: XIV) y un año más tarde es nombrado juez eclesiástico ${ }^{12}$ (Scholes y Adams, I, 1938: XLVI), año en el que es llamado a intervenir con apoyo de don Diego Quijada, alcalde mayor de Yucatán, en los casos de idolatría registrados en Maní en 1561. Parte del debate que se desarrollará a posteriori y que deriva de la intervención de Landa en las investigaciones de idolatría tiene que ver con la bula papal Exponi Nobis (Nota 12) y la Real Provisión de la Audiencia de los Confines de 28 de febrero de 1558 (Scholes y Adams, 1938, I: XVI). De estos documentos derivan los enfrentamientos posteriores que tuvieron lugar entre Landa y el obispo fray Francisco de Toral, sobre los que dan testimonio algunos autores como el cronista Diego López de Cogolludo (1955: 153) o Scholes y Adams (1938: LXI). Las relaciones entre el obispo y los franciscanos de Yucatán tampoco eran especialmente cordiales. En una carta que data de 20 de abril de $1567,{ }^{13}$ el propio Toral menciona su mala relación con ellos en la frase “..., por aver yo afeado las cosas que los religiosos

\footnotetext{
${ }^{11}$ Un contexto más amplio sobre esta cuestión puede consultarse, por ejemplo, en Raimúndez (2015), Clendinnen (2003) o González Cicero (1978).

${ }^{12}$ Hasta 1562 los asuntos eclesiásticos de Yucatán fueron realizados por los prelados de los franciscanos a falta de un obispo residente y ejercieron tal autoridad en virtud de la bula Exponi Nobis del Papa Adriano VI, fechada en Zaragoza el 10 de mayo de 1522 (Scholes y Adams, 1938, I: XV). En una carta del defensor de indios del 8 de marzo de 1563 (AHN, Diversos-Colecciones, 24, N. 64, ff. 1r-2v) se hace referencia a esta bula, sobre la que se apoyaban los frailes franciscanos para poder ejercer el derecho inquisitorial durante las investigaciones de Maní, y especialmente fray Diego de Landa para argumentar su defensa en el juicio al que fue sometido tras los hechos.

${ }^{13}$ AHN, Diversos-Colecciones, 25, N. 3, ff. 1r-2v.
} 
hizieron contra los naturales, que me tienen gran odio por ello ..." (Cartas de Indias, 1877: 243) y también hace alusión al envío de una serie de cartas: “... los religiosos, y ávn hacen escrevir a los naturales lo que no entienden, porque les dan las cartas escritas y azen las firmen, y los pobres no las entienden, y asi me an venido a dezir los naturales que an firmado lo que no entienden, y creo escrevirán a V. M. sobre ello los caciques" (Cartas de Indias, 1877: 243). Es muy probable que Toral se estuviese refiriendo a las cartas de este corpus documental.

Landa había comenzado su investigación como juez inquisitorial con el apoyo de diversos frailes, entre los que se encontraba fray Miguel de la Puebla, nombrado en las “cartas de los caciques" de Yucatán (Anexo 2, texto en maya, línea 19). Tras las investigaciones, tuvo lugar el Auto de Fe de Maní en 1562, proceso en el que fueron quemados gran cantidad de ídolos, otra parafernalia ritual y códices (Chuchiak, 2005: 31), además de condenar a los acusados de participar en los actos de idolatría, entre los que se encontraban el cacique, el gobernador, los principales y el ahkin de Maní, incluidos también algunos maestros y mozos de escuela (Lentz, 2009: 142). Las medidas de Landa durante el proceso le llevaron a ser juzgado ante el Consejo de Indias (Timmer, 1997: 479).

El obispo fray Francisco de Toral había sido nombrado en 1560 (Scholes y Adams, 1938, I: XVII), pero no fue sino hasta agosto de 1562 que llega a Yucatán, tras haber recibido las bulas papales que le otorgaban el cargo. En el momento de su llegada, los problemas derivados de las investigaciones de Maní estaban en pleno auge. Toral, informado de la situación, decidió intervenir. Modificó algunas de las decisiones que habían sido tomadas por Landa y reguló los castigos impuestos por él (Scholes y Adams, 1938, I: LXI; Restall y Chuchiak, 2002: 652).

Convocado por el Consejo de Indias, Landa regresó a España donde se le acusaba de diversos cargos. Las denuncias que le llevaron a esta situación habían provenido de diversas personas y autoridades. El obispo Toral fue uno de los encargados de denunciar a los franciscanos ante el rey; también realizaron denuncias los encomenderos, que estaban enfrentados a los religiosos por la merma de sus competencias y poder sobre los indígenas (Scholes y Adams 1938: XX); y el propio defensor de los indios, que denunciaba el mal trato dado a los indígenas en una carta de 8 de marzo de 1563, poco después de los sucesos de Maní en la que dice:

...in estas dichas provinçias a sucedido in perjuicio $\varepsilon$ gran daño de muerte. lisiones. perdidas. $\mathcal{E}$ desasosiegos de estos pobres yndios y lo que pasa [...] los frayles para que tanbien fuesen inquisidores y algunos todos $\varepsilon$ cada uno por si an hecho desatinos $\varepsilon$ castigos $\varepsilon$ n esos yndios... ${ }^{14}$

No fueron tampoco una excepción los gobernadores de Maní, que el 12 de abril de 1567 envían una carta ${ }^{15}$ a Felipe II sobre los abusos que habían cometido

\footnotetext{
${ }^{14}$ AHN, Diversos-Colecciones, 24, N. 64, ff. 1r-2v.
}

${ }^{15}$ AHN, Diversos-Colecciones, 25, N. 2, ff. 1r-2v. 
los franciscanos y las crueldades de los procesos contra las idolatrías (Bracamonte, 2001: 63). A su llegada a España, Landa tenía en su contra la predisposición del Consejo de Indias, donde se le acusaba del abuso de sus derechos como provincial, ya que se consideraba que se había extralimitado en sus funciones, de la usurpación de la jurisdicción inquisitorial, que Landa empleó a pesar de que los tribunales inquisitoriales no se habían desarrollado aún en Indias ${ }^{16} \mathrm{y}$, finalmente, de la relación con las muertes y lesiones que sufrieron los indígenas durante los procesos. En este caso se demostró el uso de castigos desmesurados y de la tortura para obtener las confesiones de los indígenas, los cuales se retractaron en el momento en el que intervino el obispo Toral (Scholes y Adams, 1938: LII). Durante el juicio, que tuvo lugar entre 1565 y $1569,{ }^{17}$ fueron realizadas y enviadas las cartas en cuestión a Felipe II, con la finalidad de apoyar a Landa durante el proceso. Aunque llegan en 1567, la decisión de indultarlo ya había sido tomada en 1565, si bien no es hasta 1569 que se da a conocer públicamente la decisión (Timmer, 1997: 479).

\section{Elaboración de las "cartas de los caciques"}

Las "cartas de los caciques" se presentan todas en el mismo formato. Se trata de un pliego en papel europeo doblado in folio enviado en forma de petición y dirigido al rey Felipe II. La filigrana se ubica en el centro del documento, y en el caso concreto de la Carta 7, la marca de agua se trata de la conocida como "filigrana del peregrino" (Figura 1), muy habitual en los documentos del siglo xvI, lo cual corrobora la autenticidad y el año de su creación. Las cartas se presentan en el verso del primer folio, con el texto en maya yucateco, y en el recto del segundo folio con el texto en castellano. Todas ellas fueron escritas entre los meses de febrero y marzo de 1567 y firmadas por los caciques yucatecos del momento. Existe una excepción a esta presentación, la Carta 5, con el primer texto en castellano y el segundo en maya yucateco. El asunto de los documentos es solicitar el envío de más frailes franciscanos a la península de Yucatán incidiendo en el regreso de los frailes Diego de Landa, Pedro Gumiel y Miguel de la Puebla, todos ellos activos partícipes de los sucesos de Maní de 1562. En el contenido de estos documentos se ponen en duda las acusaciones realizadas a los frailes y se defiende a los franciscanos sometidos a juicio, presentándolos como personas queridas y deseadas en Yucatán.

El contenido de la Carta 7 se presenta completo en los Anexos 1 y 2. Las manos intervinientes son diferentes para el cuerpo del documento en castellano y

\footnotetext{
${ }^{16}$ Los tribunales inquisitoriales fueron creados en América por medio de la Real Cédula de 25 de enero de 1569 de Felipe II, que fundaba los Tribunales del Santo Oficio en Perú y México (Medina, 1905: 33).

${ }^{17}$ AGI, Escribanía, 1009A, Pleitos del Consejo.
} 
el maya. Las firmas de los caciques que se muestran en la versión en maya son todas realizadas por la misma mano, diferente de la que escribe el cuerpo del documento. La parte correspondiente al membrete tiene un total de tres manos diferentes: una para el remitente, una para el destinatario y una para la explicación del contenido del documento. En total esta carta fue escrita por seis manos distintas. Si se analiza el contenido de ambas partes, tanto la castellana como la maya, se puede ver que son bastante aproximadas, aunque se deben tener en cuenta ciertos aspectos. En primer lugar, los paralelismos entre ambas cartas son evidentes, ya que las expresiones que aparecen en castellano y en maya yucateco son similares o calcos de una lengua a otra. Viendo el estilo de las expresiones, las que aparecen en el texto maya son tradicionalmente castellanas, con la misma forma y construcción, pero escritas en lengua maya, lo que indica que las expresiones son de origen español y por lo tanto fueron copiadas del texto en castellano: "...conforme a nuestra bajeza de ingenio y pobreza de bienes temporales..." (Anexo 2, en castellano, línea 6-7), con el texto maya: hibici cacaabalil ti vetel cahnumvailil tubahibail vokol cabe, "...por nuestra bajeza de ingenio y nuestra pobreza de cosas sobre la tierra...” (Anexo 2, en maya, línea 8).

Ambas representan la misma idea, pero viendo el análisis del documento en maya se aprecia el esfuerzo del traductor por plasmar la palabra "pobreza" derivándola de forma poco habitual. Para ello parte de la palabra numya, "pobre", empleando dos sufijos derivacionales que funcionan en el primer caso como adjetivador $(-i l)$, y en el segundo como abstractivizador (-il). De esta manera, del sustantivo "pobre" crea el adjetivo "pobre" y lo abstractiviza como "pobreza". Esto, por un lado, indica que la palabra que el escribano está empleando no es común en su escritura y, por otro lado, evidencia la existencia de un modelo sobre el que trabaja a la hora de escribir, del que toma como ejemplos estas palabras.

En la frase, además, se aprecia una expresión relacionada con el concepto de indio miserabilis. Este término, nacido del ámbito judicial, fue un concepto aplicado regularmente a mediados del siglo xvi por los religiosos yucatecos para referirse a los indígenas (Cunill, 2011: 231). En origen, el término hacía referencia al amparo de los pobres como una obligación de las autoridades civiles y eclesiásticas (Borah, 1996: 25; Ruigómez, 1988: 37-38). Pero su aplicación a los indígenas se hacía con la idea de su desventaja frente a los españoles, relacionando sus condiciones de vida con la pobreza, la ignorancia y el miedo (Cunill, 2011: 234-235). Al mismo tiempo, se vincula con el debate sobre la servidumbre natural de los indígenas que se desarrolló durante la primera mitad del siglo xvi. Dicha discusión incluía multitud de aspectos ideológicos, filosóficos y morales sobre la cuestión de los indígenas (Maestre, 2004).

Otro ejemplo que se puede ver en el texto castellano de la Carta 7 es: “...se compadezca de nuestras animas y nos envíe frailes franciscos que nos guíen y enseñen la carrera de Dios..." (Anexo 2, en castellano, línea 12) y en su versión maya ca auokecaich tacpixan ca atu[m]te ca atuxchite frailes fran[cis]cos toon pavic 
cabeel vetel cambecicoon tubelil Dios, “...que te compadezcas de nuestras almas (y) que consideres enviar frailes franciscos a nosotros (que) guíen nuestro camino y nos enseñen el camino de Dios..." ${ }^{18}$ (Anexo 3, línea 15-16). A través de la acepción $o k$, que se suele traducir como "llorar", se construye, por medio del ich- un nuevo término que significa "tener piedad". En el documento en castellano aparece la palabra "compadezca" que tiene el mismo sentido. A pesar de las pequeñas diferencias existentes entre ambas frases, la intencionalidad es la misma ya que se ha traducido la idea expresada en el texto español.

La solicitud de más frailes para la evangelización en la península de Yucatán es un tema habitual en las peticiones de esta área, aunque el formato en el que son presentadas generalmente es diferente al del corpus. Como ejemplo, se presenta una petición de origen indígena que se relaciona con las "cartas de los caciques", llamada "Carta de los gobernadores", ${ }^{19}$ que se ha denominado aquí Carta 8. En este caso no pertenece al mismo grupo de cartas debido a las amplias diferencias que existen con el resto de documentos, pero sí se puede enmarcar dentro del mismo contexto histórico (Raimúndez, 2015). La Carta 8 se realiza en 1567 al igual que el resto del corpus, se escribe en maya yucateco y es firmada por una serie de gobernadores indígenas. Las diferencias que presenta son su extensión y, debido a ello, su contenido más amplio; además, su composición dividida en tres partes y la aparición de elementos de validación junto a los nombres de las autoridades intervinientes en su producción, totalmente ausentes en las otras cartas. Uno de los aspectos que destacan del texto maya es su elaboración sin un modelo castellano, dando una libertad mayor al escribano. A diferencia de las otras cartas, carece de expresiones forzadas y literales, por lo que también permite una comparación y a su vez una reafirmación de la existencia de un modelo para la elaboración del corpus.

El autor de la traducción de la Carta 8 es Alonso de Arévalo, el intérprete general de Yucatán, nombrado para tal cargo en 1560 por el visitador García Jofre de Loayza, siendo posteriormente renombrado por el gobernador Céspedes de Oviedo en 1566. La finalidad del cargo de intérprete era la de traducir diferentes tipos de documentos escritos en lenguas indígenas para permitir una correcta comunicación entre estos y los representantes de las instituciones coloniales. (Cunill, 2015: 19).

Considerando que las fechas de las cartas son muy cercanas y las distancias entre los diferentes lugares en que fueron realizadas son relativamente amplias (Mapa 1), ello sugiere que existía un acuerdo previo por el cual se dio la orden de escribir los documentos que servirían de modelo para los escribanos que las realizaron. Su localización coincide con la disposición de las poblaciones indígenas al inicio de la Colonia. Estas organizaciones fueron las guardianías, formadas por cabeceras y visitas. Las cabeceras eran las poblaciones principales y más

\footnotetext{
18 Traducción de la autora.

${ }^{19}$ AGI, México, 359, r. 2, N. 10, ff. 223-228.
} 
importantes en torno a las cuales se organizaban las visitas, que eran pueblos dependientes de la cabecera con menor relevancia. Si se comparan los lugares de procedencia de los caciques firmantes de las cartas del corpus (Hanks, 2010: 40), la correlación entre la procedencia de éstos y las guardianías asociadas coincide. Esto apunta claramente a la creación de las cartas desde los circuitos de comunicación franciscanos (Mapa 2) y no desde los usados por los propios indígenas durante todo el periodo colonial, paralelos a ellos (Cunill, 2017; Okoshi, 2017). Existen dos cartas que no coinciden con dicho sistema organizativo, las Cartas 5 y 6, que pertenecen a las áreas de Champotón y Tixchel, respectivamente. La organización de estas áreas es diferente y menos conocida que las de las otras provincias de Yucatán (Scholes y Roys, 1996: 39), por lo que los firmantes no aparecen asociados a ninguna población y únicamente se ha podido establecer el área en torno a la que se realizaron.

Es interesante destacar la población de Tixchel, que se encontraba en la provincia de Acalán, en el área linguística chontal. La localización de la Carta 6 se pudo realizar gracias a uno de los firmantes, Pablo Paxbolón, que era el gobernante de Tixchel en esos momentos y había sido educado por frailes franciscanos en Campeche (Scholes y Roys, 1996: 148-157). En Tixchel se hablaba chontal en el siglo xvi y durante la conquista había sido escogida por los frailes como cabecera de doctrina, junto con otros cuatro pueblos, uno de ellos de lengua maya yucateca, para congregar a los chontales de Acalán, traslado que se llevó a cabo pocos años antes de la realización de estas cartas (Izquierdo, 1997: 54). Ello explica la existencia de una carta procedente del área linguiística chontal pero escrita en maya yucateco.

La organización territorial colonial que se origina con los frailes franciscanos concuerda con la distribución y localización de las cartas. Probablemente el original fue una plantilla o texto modelo que fue repartida en las poblaciones afines a ellos. Al mismo tiempo los esfuerzos de los escribanos en la plasmación de las fórmulas y expresiones castellanas en los documentos escritos en maya sugieren que precisamente este modelo se distribuyó en castellano, idea que se sustenta por la ausencia de diferencias entre los textos castellanos de las cartas y por una traducción literal al maya de las expresiones propias castellanas. El hecho de que las versiones de las cartas escritas en maya sí presenten, en cambio, diferencias, apunta a la versión castellana, no como la traducción, sino como el modelo de partida.

\section{Conclusión}

La introducción de una nueva metodología crítica para el estudio de la documentación escrita en lenguas indígenas, ejemplificada en la traducción y análisis que se presenta en los anexos, ha permitido mostrar una serie de nuevas perspectivas sobre las "cartas de los caciques". Entre ellas, el estudio integral del documento, la revisión del texto escrito en maya y una nueva propuesta de traducción. 


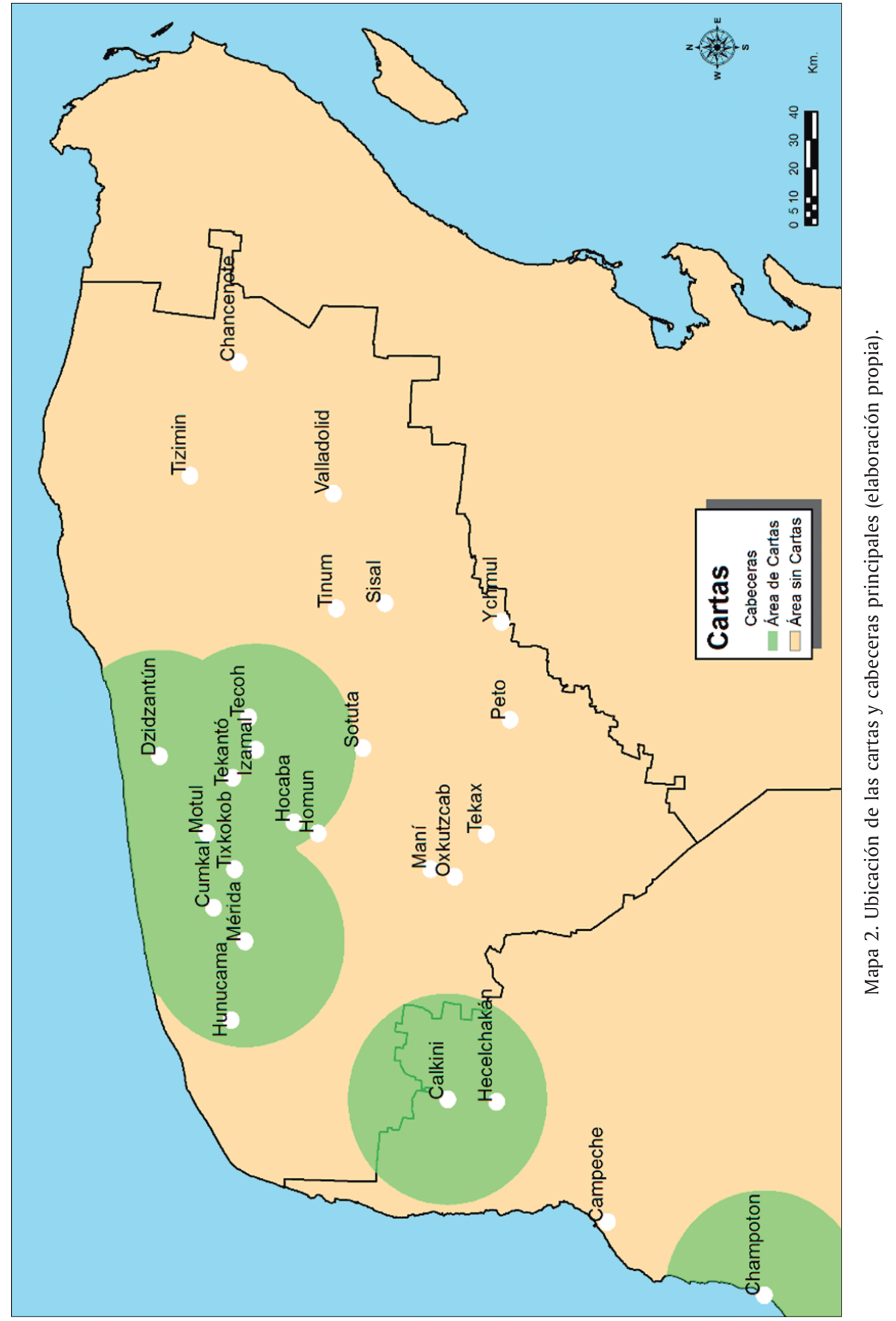


El trabajo de archivo realizado durante la investigación ha supuesto el descubrimiento de información hasta ahora desconocida, permitiendo la aportación de la Carta 7 como un documento inédito que abre nuevas posibilidades de estudio y ampliación de este grupo de cartas. Por un lado, comparar el texto en castellano con la traducción del maya permite ver la probable redacción de un modelo de documento escrito en castellano por parte de los franciscanos, ya que se puede apreciar la existencia de calcos y traducciones literales. La intervención franciscana da lugar a la creación de este corpus con unos intereses específicos y aconseja revisar otros documentos que presentan el mismo formato. Por otro, la inserción de estas cartas en la discusión más amplia sobre el concepto de indio miserabilis aumenta el alcance del contenido y abre una interesante vía de investigación en su aplicación a otros textos indígenas.

La Carta 8 presenta un modelo de documento que se puede contraponer claramente al resto, ya que la traducción es mucho menos coherente y clara que la de las "cartas de los caciques", esto pone de manifiesto las diferencias en los procesos de elaboración de las "cartas de los caciques" y la de la carta de los gobernadores. Su contexto como documentos vinculados a los primeros años de la historia de Yucatán y la importancia de la información a la que se refieren, relacionada con el Auto de Fe de Maní y el juicio de Landa, además de otros aspectos como la organización territorial, el concepto de "indio miserable" o matices linguíísticos particulares, se han podido inferir a través de la aplicación de esta metodología, que puede ser utilizada en el estudio de otros documentos similares.

\section{Bibliografía}

Arzápalo, Ramón (ed.)

1995 Calepino de Motul, tomo III. México: Universidad Nacional Autónoma de México, Instituto de Investigaciones Antropológicas.

Barrera Vásquez, Alfredo (coord.)

1980 Diccionario maya-español, español-maya. Mérida: Cordemex.

Batalla, Juan José

2008 "Los códices mesoamericanos: métodos de estudio", Itinerarios, 8: 43-65.

Bracamonte y Sosa, Pedro

2001 La conquista inconclusa de Yucatán. Los mayas de la montaña, 1560-1680. México: Centro de Investigaciones y Estudios Superiores en Antropología Social (Colección Peninsular).

Borah, Woodrow

1996 El Juzgado General de Indios en la Nueva España. México: Fondo de Cultura Económica (Sección de Obras de Historia). 
Cartas de Indias

1877 Madrid: Ministerio de Fomento de España.

Chuchiak, John F.

2005 "El regreso de los Autos de Fe: fray Diego de Landa y la extirpación de idolatrías en Yucatán, 1573-1579”, Península, I (0): 29-47.

Clendinnen, Inga

2003 Ambivalent Conquest: Maya and Spaniard in Yucatan, 1517-1570. Cambridge: Cambridge University Press.

Cunill, Caroline

2011 "El indio miserable: nacimiento de la teoría legal en la América colonial del siglo xv1", Cuadernos Intercambio, 8 (9): 229-248.

2012 Los defensores de indios de Yucatán y el acceso de los mayas a la justicia colonial, 1540-1600. México: Universidad Nacional Autónoma de México, Centro Peninsular en Humanidades y Ciencias Sociales.

2015 "Justicia e interpretación en sociedades plurilinguies: el caso de Yucatán en el siglo xvı", Estudios de Historia Novohispana, 52: 18-28. DOI: 10.1016/j. ehn.2014.03.001

2017 "Intercambios epistolares y conformación de espacios políticos mayas supracomunitarios en el Yucatán del siglo XVI”, Indiana, 34 (2): 35-54. DOI 10.18441/ind.v34i2.35-54

Farris, Nancy

1991 La sociedad maya bajo el dominio colonial, la empresa colectiva de la supervivencia. Madrid: Alianza Editorial.

González Cicero, Stella María

1978 Perspectiva religiosa en Yucatán, 1517-1571: Yucatán, los franciscanos y el primer obispo fray Francisco de Toral. México: El Colegio de México.

Hanks, William F.

2010 Converting Words. Maya in the Age of the Cross. Berkeley: University of California Press.

Izquierdo, Ana Luisa

1997 Acalán y la Chontalpa en el siglo xvi: su geografía política. México: Universidad Nacional Autónoma de México, Instituto de Investigaciones Filológicas, Centro de Estudios Mayas.

Lentz, Mark

2009 "Los intérpretes generales de Yucatán: hombres entre dos mundos”, Estudios de Cultura Maya, XXXIII: 135-158. DOI: 10.19130/iifl.ecm.2009.33.44

Lockhart, James

1992 The Nahuas after the Conquest: A Social and Cultural History of the Indians of Central Mexico, Sixteenth Through Eighteenth Centuries. Stanford: Stanford University Press. 
López de Cogolludo, Diego

1955 Historia de Yucatán, tomo II. Campeche: Comisión de Historia.

Maestre Sánchez, Alonso

2004 "Todas las gentes del mundo son hombres. El gran debate entre fray Bartolomé de las Casas (1474-1566) y Juan Ginés de Sepúlveda (1490-1573”, Anales del Seminario de Historia de la Filosofía, 21: 91-134.

Medina, José Toribio

1905 Historia del Tribunal del Santo Oficio de la Inquisición en México. México: Ediciones Fuente Cultural.

Okoshi Harada, Tsubasa

2017 "Espacio, tiempo y escritos: los títulos de tierras y la red de comunicación entre los pueblos de indios de Yucatán”, Indiana, 34 (2): 15-33. DOI 10.1844/ ind.v34i2.15-33

Pottier, Bernard

1969 Gramática del español. Madrid: Ediciones Alcalá.

Quezada, Sergio

1993 Pueblos y caciques yucatecos, 1550-1580. México: El Colegio de México.

2014 Maya Lords and Lordship. The Formation of Colonial Society in Yucatán, 13501600. Norman: University of Oklahoma Press.

Raimúndez, Zoraida

2015 "Las cartas de los caciques de Yucatán a Felipe II de 1567", trabajo de fin de Máster en Historia y Antropología de América. Madrid: Universidad Complutense de Madrid, Departamento de Historia de América II (Antropología de América).

Real Academia Española

2016 Diccionario de la lengua española, 23a edición. < http://dle.rae.es/> [Consultado el 26 de abril de 2016].

Restall, Mathew

1997 The Maya World: Yucatec Culture and Society, 1550-1850. Stanford: Standford University Press.

2001 "Filología y etnohistoria. Una breve historia de la 'nueva filología' en Norteamérica”, Desacatos, 7: 85-102. DOI: 0.29340/7.1399

Restall, Mathew y John F. Chuchiak

2002 "A Reevaluation of the Authenticity of fray Diego de Landa's Relación de las Cosas de Yucatán", Etnohistory, 49 (3): 651-669. DOI:10.1215/00141801-49-3651

Rojas, José Luis de

2008 La etnohistoria de América. Los indígenas, protagonistas de su historia. Buenos Aires: SB. 
Ruigómez, Carmen

1988 Una política indigenista de los Habsburgo: el protector de indios en el Perú. Madrid: Ediciones Cultura Hispánica.

Scholes, France V. y Eleanor B. Adams

1938 Don Diego Quijada alcalde mayor de Yucatán, 1561-1565, tomo I. México: José Porrúa e Hijos.

Scholes, France V. y Ralph L. Roys

1996 Los chontales de Acalán-Tixchel. México: Universidad Nacional Autónoma de México, Instituto de Investigaciones Filológicas, Centro de Estudios Mayas, Centro de Investigaciones y Estudios en Antropología Social.

Timmer, David

1997 "Providence and Perdition: Fray Diego de Landa Justifies his Inquisition against the Yucatecan Maya", Church History, 66 (3): 477-488. DOI $0.2307 / 3169452$

Von Zimmerman, Günter

1970 Briefe der Indianischen Nobilität aus Neuspanien an Karl V und Philipp II um die Mitte des 16. Hamburgo: Herausgegeben vom Hamburgischen Museum für Völkerkunde und Vorgeschichte.

\section{Documentos de archivo}

Ministerio de Cultura y Deporte. Archivo General de Indias (Sevilla, España)

AGI, Escribanía de Cámara, Legajo 1009A, Pleitos del Consejo "Fray Francisco de Toral, obispo de Yucatán, con fray Diego de Landa, provincial de la Orden de San Francisco en dicha provincia, sobre haber ejercitado el oficio de inquisidor e invocado el brazo seglar para castigar a los indios idólatras". Campeche, 1585, ff. 1r-1v.

AGI, México, Legajo 359, Caja 2, Número 10, “Carta de los Gobernadores de Yucatán”, Mérida, 1567, ff. 223-228.

AGI, México, Legajo 367, “Carta de los caciques de Yucatán”, Yucatán, 1567, ff. 62r-63v.

AGI, México, Legajo 367, “Carta de los caciques de Yucatán”, Yucatán, 1567, ff. 64r-65v.

AGI, México, Legajo 367, “Carta de los caciques de Yucatán”, Yucatán, 1567, ff. 66r-67v.

AGI, México, Legajo 367, “Carta de los caciques de Yucatán”, Yucatán, 1567, ff. 68r-69v.

AGI, México, Legajo 367, “Carta de los caciques de Yucatán”, Yucatán, 1567, ff. 70r-71v.

AGI, México, Legajo 367, “Carta de los caciques de Yucatán”, Yucatán, 1567, ff. 88r-89v. Archivo Histórico Nacional (Madrid, España) 
AHN, Diversos-Colecciones, caja 24, número 64, "Carta solicitando la expulsión de franciscanos de Yucatán”, Mérida, 1563, ff. 1r-2v.

AHN, Diversos-Colecciones, caja 24, número 77, "Carta de fray Francisco de Toral”, México, 1565.

AHN, Diversos-Colecciones, caja 25, número 2, "Carta de los gobernadores de Maní”, Maní, 1567, ff. $1 \mathrm{r}-2 \mathrm{v}$.

AHN, Diversos-Colecciones, caja 25, número 3, "Quejas sobre el gobernador de Yucatán”, Mérida, 1567, ff. 1r-2v.

Zoraida Raimúndez Ares. Española. Licenciada en Historia y maestra en Historia y Antropología de América por la Universidad Complutense de Madrid. Actualmente realiza estudios de doctorado en el Posgrado de Estudios Mesoamericanos de la Universidad Nacional Autónoma de México y en Historia y Arqueología en la Facultad de Geografía e Historia de la Universidad Complutense de Madrid. Sus intereses académicos se centran en la etnohistoria e historia de los mayas coloniales y su proyecto actual de investigación se titula "Difrasismos en los textos mayas yucatecos coloniales". Participa en el Proyecto "Cuerpo y simbolismo amerindio: diseño museográfico y docente de la colección complutense del Museo de Arqueología y Etnología de América referida al cuerpo humano”, dirigido por el Dr. Alfonso Lacadena García-Gallo (†). Entre sus contribuciones puede mencionarse "Un acercamiento a los documentos coloniales en maya yucateco a través del método codicológico, paleográfico y diplomático”.

zraiare@hotmail.com zraimund@ucm.es 


\section{Anexo 1. Facsímil de la Carta 7. Ministerio de Cultura y Deporte. AGI, México, Legajo 367, folios 88r-89v.}

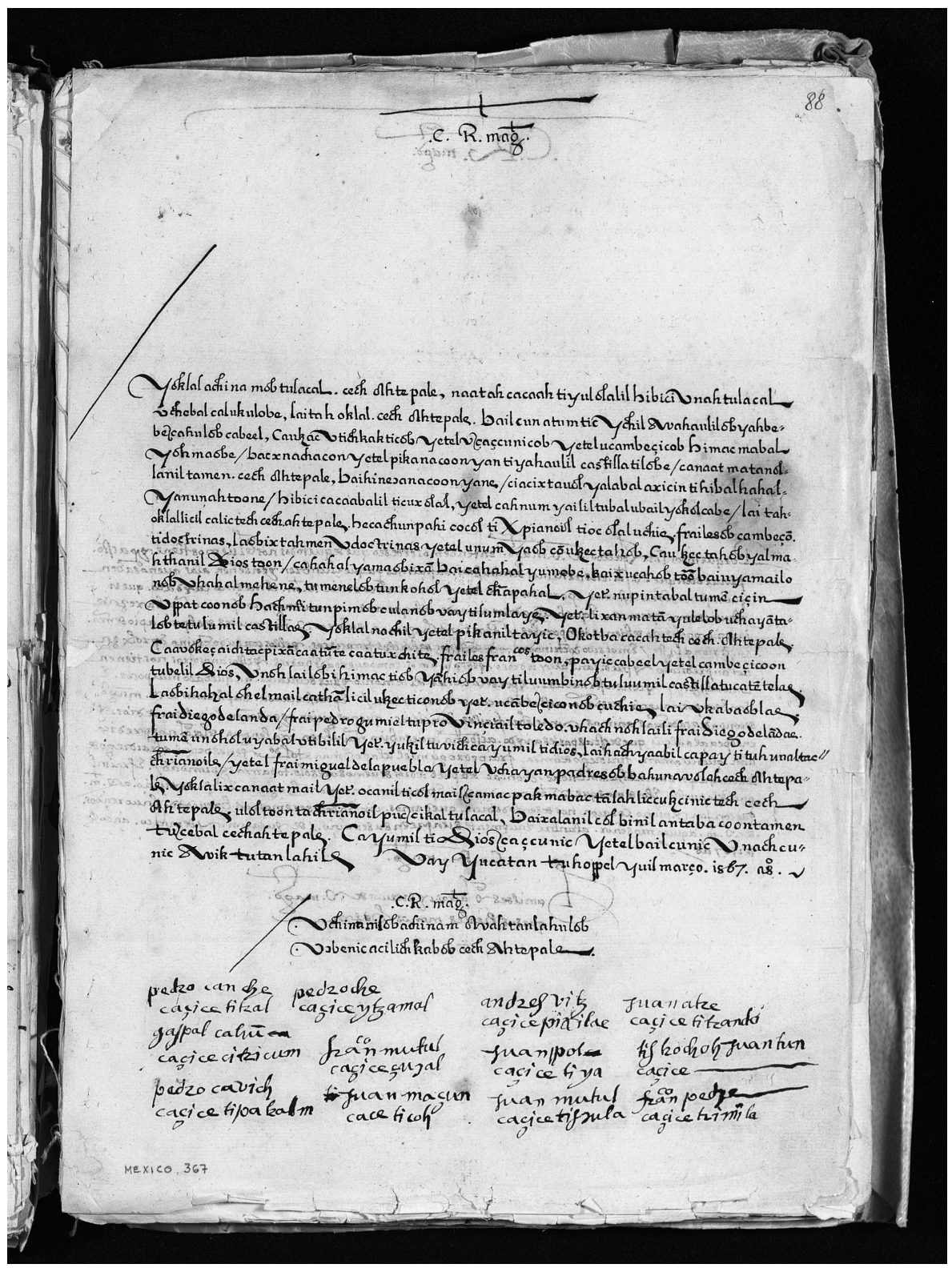




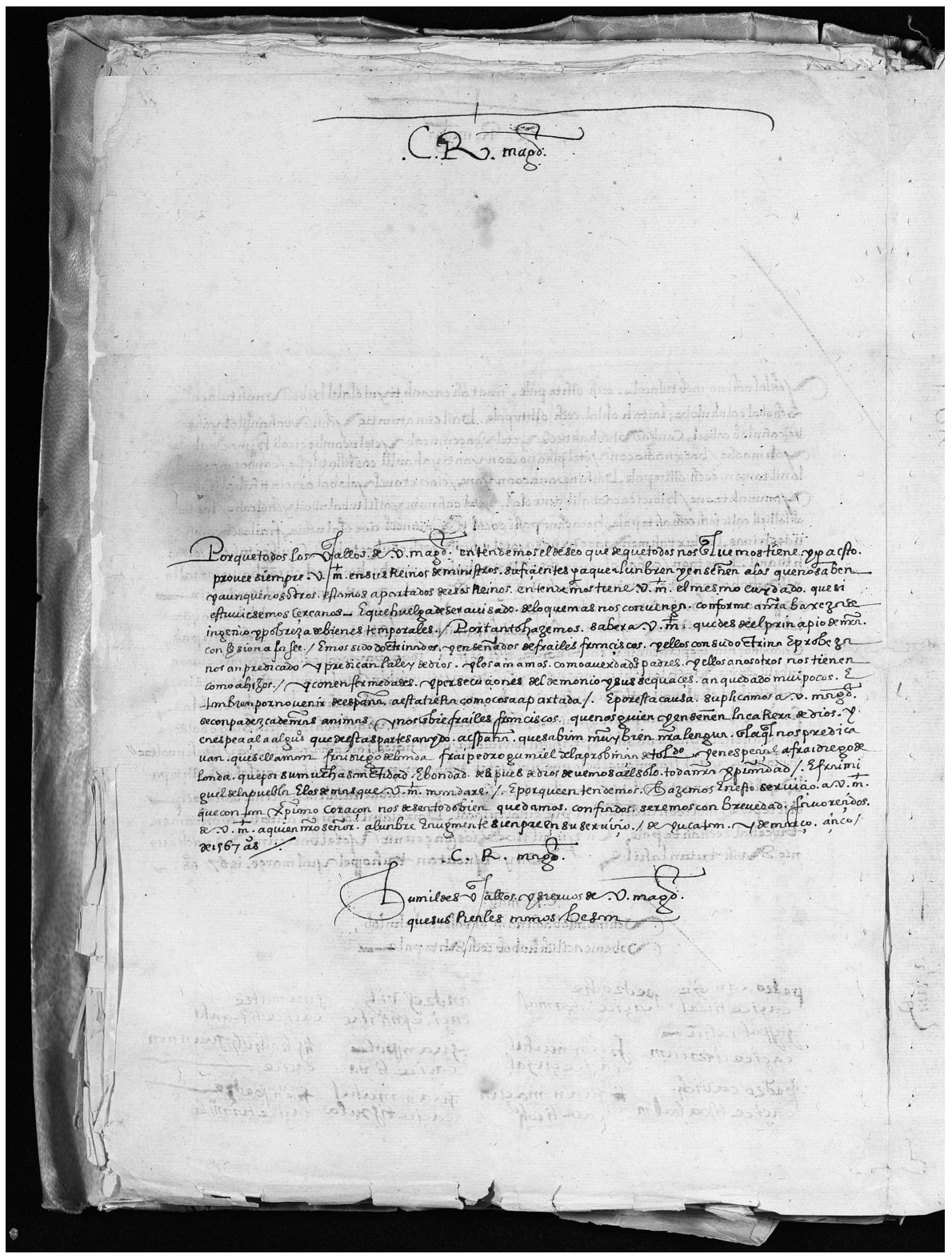




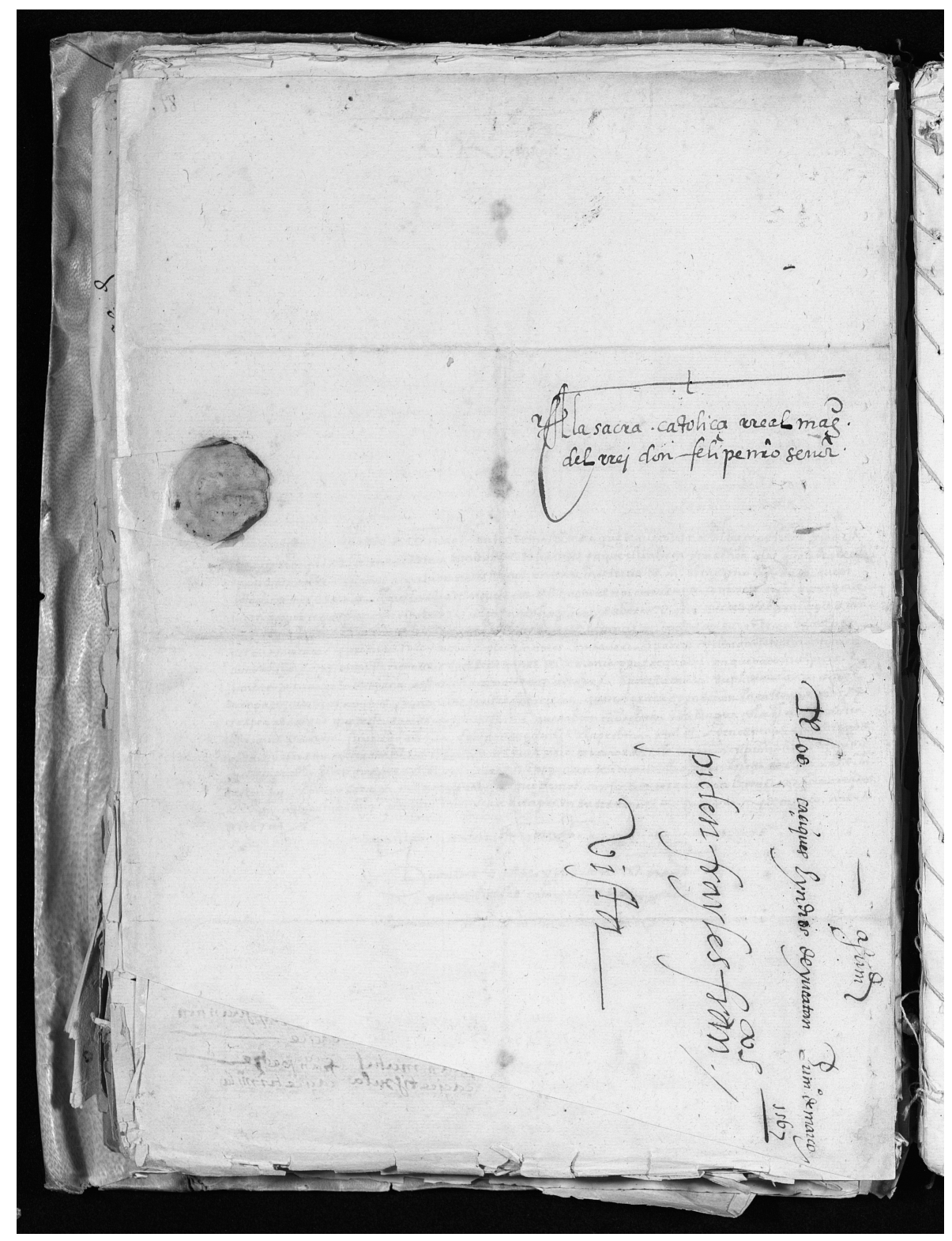


Anexo 2. Transliteración de la Carta 7.

$1 /$

2 $\dagger$

3/Yoklal achinamob tulacal. cech ahtepale, naatah cacaah ti yulolalil hibici Unah tulacal

4/uchebal calukulobe, lai tahoklal. cech ahtepale. bailcun atumtic Ychil Auahaulilob yahbe//

5/beçahulob cabeel, Ca utzac Utichkakticob Yetel uçaçcunicob Yetel ucambeçicob himac mabal

6/Yohmaobe/ bacx nachacon Yetel pikanacoon yan ti yahaulil castilla tilobe/ canaat matan ol.

${ }^{7}$ /lanil tamen. cech ahtepale, baihi neoanacoon yane/ciacix tauol Ylabal axicin ti hibal hahal.

8/Yan unah toone/ hibici cacaabalil ti cux olal, yetel cahnumyailil tubalubail yokol cabe/ lai tah

9/oklal licil calic tech cech ahtepale, he cachunpahic ocol ti Xpianoil ti ocolal uchie, frailesob cambeçō.

${ }^{10} /$ ti doctrinas, laobix tahmen Udoctrinas yetel unum Yaob cō ulgectahob, Ca ulgectahob yalma

11/hthanil Dios toon/ cahahalyamaobi xā Vai cahahal yumobe. baix ucahob tōō bai uyamailo

12/nob Uhalal mehene, tumenelob tun kohol Yetel chapahal. Yet' nupintabal tumē ciçin

13/ Urpatcoonob hach $\mathrm{m}^{\mathrm{a}} \mathrm{i}$ tun pimob culanob uay ti lum laye. Yet'.Li xan matā yulelob uchaya tā//

14/Iob te tulumil castillae Yoklal nachil yetel pikanil tayic, Okotba cacah tech cech ahtepale

15/Ca auokeçaich tacpixā ca atūte ca atuxchite. frailes fran ${ }^{\cos }$ toon, payic cabeel yetel cambeçicoon

15/tubelil Dios, Unohlailobi himac tiob Yāhiob uay ti luum binob tuluumil castilla tucatē te lae

16/ Laobi hahal ohel mail cathā licil ubecticonob yet'. ucābeçiconob cuchie lai -Ukabaob lae

${ }^{17}$ /frai diego de landa/frai pedro gumiel tuproVinçiail toledo. uhach nohlaili frai diego de lādae

18/tumē unohol uyabal utibilil Yet'. yutgil tuuich cayumil ti dios, lai hach yaabil capay ti tuhunal tac//

${ }^{19} /$ chrianoile/ yetel frai miguel de la puebla Yetel Uchayan padresob bahun auolah cech ahtepa

20/le. Yoklalix canaat mail Yet' ocanil ti col mail çamac pakmabac tālah lic culgcinic tech cech 
${ }^{21}$ /Ahtepale uloltoon tachrianoil pucçikal tulacal, Baix alanil col binil antabacoon tamen

${ }^{22}$ /tuçebal cech ahtepale Cayumil ti Dios çaçcunic Yetel bailcunic Unachcury

${ }^{23} /$ nic Auik tutanlahil Uay Yucatan tuhorpel yuil março. 1567. as ${ }^{\circ}$.

24/ C. R. mag $^{+}$.

25. Uchimamilob achinam auahtanlahulob

26/ Usbenic acilich kabob cech Ahtepale

${ }^{27}$ /pedro canche pedro che andres vitz juan atze

${ }^{28}$ /caçice titzal caçice ytzamal caçice pixilae caçice titzanki

${ }^{29} / \mathrm{ga}$ jpal cahu ea fran ${ }^{\text {co }}$ mutul juan polea lif hochoh juan tun

${ }^{30}$ /caçice citzicum caçice çůal caçice tiya caçice

${ }^{31}$ /pedro cauich ti juan maçun juan mutul fran ${ }^{\mathrm{co}}$ pech

32 / caçice tipakalm cace ticoh ${ }^{\circ}$ caçice tifoula caçice tzimila // fol 1r.

$1 /$
$2 /$$\quad$ C. R. $\operatorname{magd}^{+}$.

3/ Porque todos los vāllos de v. magd ${ }^{+}$. entendemos el deseo que de que todos nos salvemos tiene y pa efto.

${ }^{4} /$ prouee siempre. $v . \mathrm{m}^{+}$. en sus Reinos de ministros. sufiçientes pa que alunbren y enseñen a los que no saben

5/y aunque nosotros. eftamos apartados de esos Reinos entendemos tiene v. $\mathrm{m}^{+}$. el mesmo cuidado que si

${ }^{6} /$ eftuviesemos çercanos_E que huelga de ser avisado de lo que mas nos convenga. conforme a n̄rā baxeza de

${ }^{7}$ /ingenio y pobreza de bienes temporales/Por tanto hazemos saber a v. $\mathbf{m}^{+}$. que desde el principio de ñrā.

${ }^{8} /$ con§sion a la fee/ Emos sido doctrinados. y enseñados de frailes françiscos. y ellos con su doctrina $\varepsilon$ probeza

$9 /$ nos an predicado $\mathrm{Y}$ predican la ley de dios. y los amamos. como a uerdad $^{\circ} \mathbf{s}$ padres. y ellos a nosotros nos tienen

${ }^{10} /$ como a hijos/ Y con enfermedades. y persecuçiones del demonio y sus sequaçes. an qedado mui pocos. $\varepsilon$

${ }^{11} / \tan$ bien por no uenir de españa a efta tieRa como cosa apartada/ $\mathcal{E}$ por efta causa. suplicamos a v. magd $^{+}$.

${ }^{12}$ se conpadezca de ñrās animas y nos èbie frailes franciscos que nos guien y enseñen la caRera de dios. $Y$

${ }^{13} /$ en espeçial a $\operatorname{algu}^{\circ}$ s que de eftas partes an ydo a efpaña. que sabian mūy bien. n̄rā lengua. $\bar{e}$ la $\bar{q} \overline{\text { n}}$ nos predica

14/uan. que se llaman frai diego de landa frai pedro gumiel de la provinçia de toldo y en espeçial a frai diego de

${ }^{15} /$ landa. que por su mucha sanctidad $\mathcal{E}$ bondad despues de dios deuemos a el solo toda n̄rā xpīāndad $/ . \varepsilon$ frai mi 
${ }^{16}$ /guel de la puebla $\mathcal{E}$ los demas que $\mathrm{v}^{+} \mathrm{m}^{+}$. mandare./ $\mathcal{E}$ porque entendemos Hazemos $\varepsilon$ n efto servicio a. v. $\mathrm{m}^{+}$.

${ }^{17}$ / que con tan xpīāno coraçon nos desea todo bien quedamos. confiados. seremos con brevedad. favoreçidos.

${ }^{18} /$ de. $v . \mathrm{m}^{+}$. a quien ñ̄̃o señor. alunbre $\mathcal{E}$ augmente sienpre en su serviçio./de yucatan. y de março. çinco/

$19 /$ de 1567 as $\%$

$20 /$

C. R. magd $^{+}$.

21/

$22 /$

humildes vāllos. y siervos de. V. magd $^{+}$.

que sus Reales manos besan// fol 1v. 


\section{Anexo 3. Transcripción, análisis y traducción de la Carta 7}

Fol. 1r.

$1 / \uparrow$

(cruz)

(cruz)

²C[atólica] R[ea]l Mag[estad]

"Católica Real Majestad"

3/Yoklal achinamob

yokal a-chinam-ob

tulacal,

cech

Ahtepale, ${ }^{20}$

porque 2sE ${ }^{21}$-vasallo ${ }^{22}$-PL PREP-3sE-todo

c-ech ah-tepal-e

"Porque todos tus vasallos, tú que eres el Rey, ${ }^{23}$

PRON-2sA

AG-rey-CLIT

$\begin{array}{llll}\text { naatah } & \text { cacaah } & \boldsymbol{t i} & \text { yulolalil } \\ \text { naat-ah } & \text { ca-caah } & \text { ti } & \text { y-ul-ol-al-il } \\ \text { entender-APAS } & \text { 1pE-INCdec } & \text { PREP } & \text { 3sE-llegar-corazón-SUST-REL } \\ \text { entendemos el deseo } & & \end{array}$

$\begin{array}{lllll}\text { hibici } & \text { unah } & \text { tulacal } & \text { 4/ucheba } & \text { calukulobe, } \\ \text { hi-bic- } i & \text { u-nah } & \text { ti-u-lacal } & \text { uchebal } & \text { ca-luk-ul-ob-e } \\ \text { IND-como-CLIT } & \text { 3sE-necesario PREP-3sE-todo } & \text { para que } & \text { 1pE-salvar-INC-3pA-CLIT } \\ \text { de cómo es necesario para todos que nos salvemos, } & \end{array}$

${ }^{20}$ El uso de "tú" para referirse al rey, se utiliza en todo el documento debido a que no existe una diferenciación clara entre el "tú" y el "usted" en los textos. Se ha considerado mantener el trato informal ya que, si se utilizara en este caso, sería necesario utilizarlo en todas las segundas personas en singular que aparecieran en el resto del texto, lo que implicaría un uso incorrecto del tratamiento.

${ }^{21}$ Las abreviaturas empleadas en esta traducción son 1/2/3, Primera/Segunda/Tercera Persona; A, Pronombre Absolutivo; ABL, Ablativo; ABS, Abstractivizador; ADJ, Adjetivador; AG, Agentivo; APAS, Antipasivo; CAUS, Causativo; CLIT, Clítico; COM, Completivo; dec, Modo Declarativo; DUR, Durativo; E, Pronombre Ergativo; IND, Indeterminado; INC, Incompletivo; INCO, Incoativo; Ø, No presenta forma; P, Pronombre Personal; $\mathrm{P}_{2}$, variante Pronombre Personal; PART, Partícula; PAS, Pasivo; PL, Plural; PREP, Preposición; PRON, Pronombre Relativo; PTCP, Participio; REFL, Reflexivo; Rel, Relacional; s/p, Singular/ Plural; SUB, Subjuntivo; TRANS, Transitivizador.

${ }^{22}$ La entrada (ah) chinam se define en los diccionarios, entre otras acepciones, como "señor de vasallos" (Arzápalo, 1995: 1493; Barrera, 1980: 101) y "pueblo" (Barrera, 1980: 101). En el texto en castellano aparece el término "vasallos" (Anexo 2, castellano, línea 3). La elección de este último término se entiende de la misma forma que los términos ajaw, "señor", y y-ajaw, "señor de" o "vasallo", del periodo Clásico, contraria a la concepción española de señor, que es superior al gobernado y no al contrario, como sucede en maya. Ambas consideraciones se han utilizado para justificar el empleo del término "vasallo" en la traducción.

${ }^{23}$ Las normas de traducción explican que los términos en lengua indígena relacionados con cargos o nombres propios y de lugares no se deben traducir. Debido a que se refiere específicamente a un cargo español y no indígena, se ha optado por la traducción "Rey" en lugar del término que aparece en el documento en castellano "Majestad" (Anexo 2, castellano, línea 3) debido a que aparece este mismo término escrito en castellano en el documento (Anexo 2, castellano, línea 1). Para evitar confusión entre ambas palabras, se ha elegido el término "Rey" para todos los casos en los que aparece ahtepal. 


$\begin{array}{lllll}\text { lai } & \text { tahoklal, } & \text { cech } & \text { Ahtepale, } & \text { bailcun } \\ \text { lay } & \text { ti-ca-oklal } & \text { c-ech } & \text { ah-tepal-e } & \text { bail-cun- } \emptyset \text { - } \emptyset \\ \text { DEM } & \text { PREP-1pE-Rel } & \text { PRON-2sA } & \text { AG-rey-CLIT } & \text { disposición-CAUS-SUB-3sA }\end{array}$

por nosotros, tú que eres el Rey, perseveras

atumtic

a-tum-t-ic- $\varnothing$

2sE-considerar-TRANS-COMdec-3sA

(y) consideras dentro de tus reinos ychil

chil

dentro auahaulilob

au-ahau-lil-ob

2sE-señor-ABS-PL

\section{yahbe $e^{5 / b e c ̧ a h u l o b ~ c a b e e l ~}$}

y-ah-bebeçah-ul-ob ca-beel

3pE-AG-guiar-SUST-3pE 1pE-camino

a los guías ${ }^{24}$ de nuestro camino

$\begin{array}{llll}\text { ca ubac } & \text { utichkakticob } & \text { yetel } & \text { uçaçcunicob } \\ \text { ca ubac } & \text { u-tich-kak-t-ic-ob } & \text { y-etel } & \text { u-çaç-cun-ic-ob } \\ \text { para que } & \text { 3sE-alzar-fuego-TRANS-SUB-3pA } & \text { 3sE-con } & \text { 3sE-iluminar-CAUS-SUB-3pA } \\ \text { para que alumbren e iluminen } & \end{array}$

$\begin{array}{lllll}\text { yetel } & \text { ucambeçicob } & \text { himac } & \text { mabal } & \text { 6/yohmaobe, } \\ \text { y-etel } & \text { u-camb-eç-ic-ob } & \text { hi-mac } & \text { ma-bal } & \text { y-oh-ma-ob-e } \\ \text { 3sE-con } & \text { 3sE-enseñar-CAUS-SUB-3pA } & \text { IND-quien } & \text { no-cosa } & \text { 3sE-saber-PERF-3pA-CLTT } \\ \text { y enseñen a quienes nada saben, } & & & \end{array}$
$\operatorname{bac}[i] x$
nachacon
yetel
pikanacoon
bacix
nach-ac-on
y-etel
pikan-ac-oon
puesto que lejos-SUB-1pA 3 sE-con
apartar-SUB-1 pA

puesto que estamos alejados y estamos apartados

$\begin{array}{llll}\text { yan } & \boldsymbol{t} i & \text { yahaulil } & \text { Castilla, } \\ \text { yan }^{26} & \text { ti } & \text { y-ahau-lil } & \text { Castilla } \\ \text { estar } & \text { PREP } & \text { 3sE-señor-ABS } & \text { Castilla } \\ \text { (de los que) están en el reino de Castilla, }\end{array}$

\footnotetext{
${ }^{24}$ Se ha utilizado el término literalmente, ya que es una adaptación que realiza el escribano para hacer referencia a "ministros" (Anexo 2, castellano, línea 4).

${ }^{25}$ Ambos verbos forman un recurso literario denominado parasinonimia, por el cual dos unidades cuyas sustancias de significado son parecidas forman una relación (Pottier, 1969: 146). El uso de este recurso por parte del escribano es habitual a lo largo del documento, ya que en el texto en castellano (Anexo 2, castellano, línea 4) aparece un único verbo.

${ }^{26}$ Duplica la $\mathbf{I}$ inicial que se encuentra en la línea anterior. Se ha corregido en la transcripción.
} 


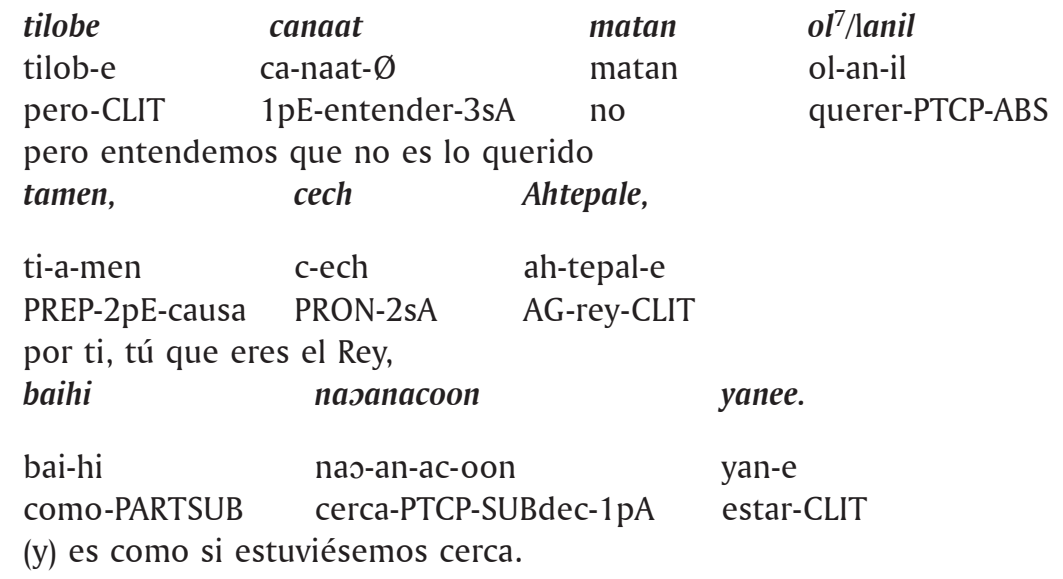

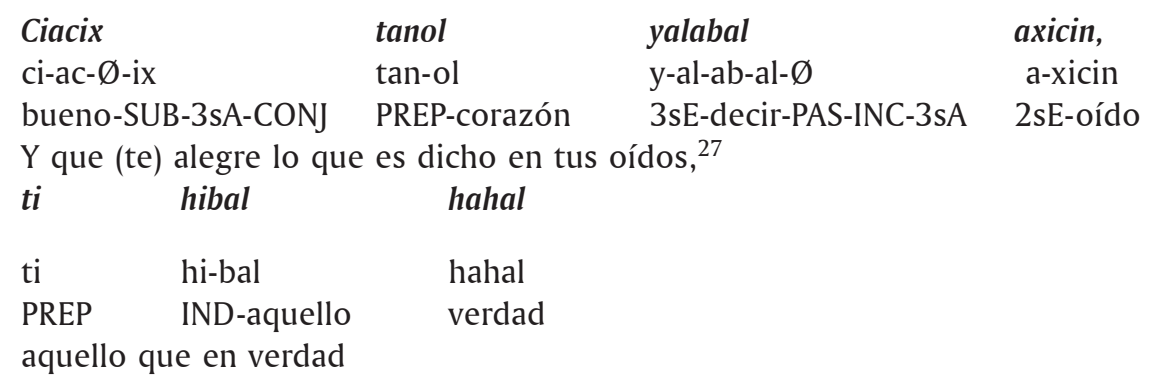

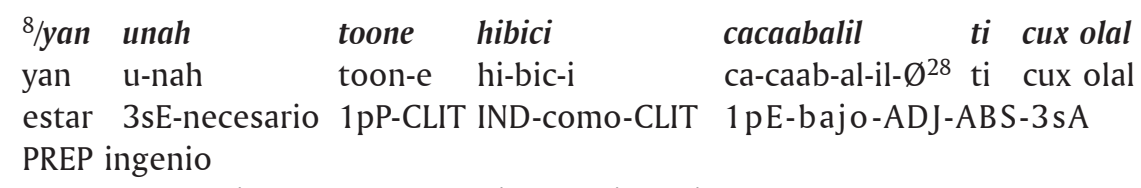

nos es necesario, por nuestra bajeza de ingenio

yetel cahnumyailil tubahibail yokol cabe,

y-etel ca-ah-numya-il-il2 ${ }^{29}$ ti-u-bahi-ba-il y-okol cab-e 3sA-con 1pE-AG-pobre-ADJ-ABS PREP-3sE-cosa-CAUS-ABS 3sE-sobre tierra-CLIT y nuestra pobreza de cosas sobre la tierra,

\footnotetext{
${ }^{27}$ Esta expresión, que equivale a "huelga de ser avisado" (Anexo 2, castellano, línea 6), es habitual en los documentos castellanos del siglo xvı. El escribano ha utilizado el sentido literal de los términos para trasladarlos al documento en maya yucateco, para ello emplea "alegre", que es el sentido original de "huelga" y "lo que es dicho a tus oídos" para sustituir "de ser avisado". Este ejemplo sostiene la idea de un modelo que el autor del texto utiliza para copiar tales expresiones.

${ }^{28}$ La traducción literal de esta palabra es "tierra" (Arzápalo, 1995, III: 1526; Barrera, 1980: 227). Las adaptaciones necesarias que ha tenido que realizar el escribano para que dicha palabra fuera similar al castellano "bajeza" (Anexo 2, castellano, línea 6) indican que el término que necesita no existe en su lengua y la necesidad de adaptar la palabra es condicionada por el modelo que emplea.

${ }^{29}$ La formación de este término sufre el mismo proceso que se explica en la nota anterior.
} 


$\begin{array}{lllllll}\text { lai } & \text { tah }{ }^{9} / \text { oklal } & \text { licil } & \text { calic } & \text { tech, } & \text { cech } & \text { Ahtepale, } \\ \text { lay } & \text { ti-ca-okal } & \text { licil } & \text { ca-al-ic- } \varnothing & \text { ech } & \text { c-ech } & \text { ah-tepal-e } \\ \text { DEM } & \text { PREP-1pE-para } & \text { INC 1pE-decir-INC-3sA } & \text { 2sP } & \text { PRON-2sA } & \text { AG-rey-CLIT } \\ \text { en lo referente a nosotros te decimos a ti, tú que eres el Rey, }\end{array}$

$\begin{array}{llll}\text { he } \quad \text { cachunpahic } & \text { ocol } & \boldsymbol{t i}^{30} & \text { cristianoil, } \\ \text { he ca-chun-pah-ic- } Q & \text { oc-ol } & \text { ti } & \text { cristiano-il } \\ \text { FOC 1pE-empezar-INTR-COM-3sA } & \text { entrar-corazón-SUST } & \text { PREP } & \text { cristiano-ABS } \\ \text { (que) habíamos empezado a creer en el cristianismo, } & & \end{array}$

ti ocolal

ti oc-ol-al ${ }^{31}$

PREP entrar-corazón-ABS

en la fe. uchie.

uchi-e

antiguamente-CLIT

$\begin{array}{llll}\text { frailesob } & \text { cambeç[oon] } & 10 / t i & \text { doctrinas, } \\ \text { frailes-ob } & \text { camb-eç- } \varnothing \text {-oon } & \text { ti } & \text { doctrinas } \\ \text { frailes-PL } & \text { aprender-CAUS-SUB-1pA } & \text { PREP } & \text { doctrinas }\end{array}$

Los frailes nos enseñan en sus doctrinas,

$\begin{array}{lll}\text { laobix } & \text { tahmen } & \text { udoctrinas } \\ \text { laob-ix } & \text { ti-ah-men } & \text { u-doctrinas } \\ \text { DEM-CONJ } & \text { PREP-AG-maestro } & \text { 3sE-doctrinas } \\ \text { y estos maestros con sus doctrinas } & \end{array}$

$\begin{array}{llcl}\text { yetel } & \text { unumyaob } & \text { co[on] } & \text { ulzectahob } \\ \text { y-etel } & \text { u-numya-ob } & \text { coon } & \text { u-bect-ah-ob } \\ \text { 3sE-con } & \text { 3pE-pobreza-3pE } & 1 \mathrm{pP} 2 & \text { 3sE-predicar-TRANS-COM-3pA } \\ \text { y su pobreza nos predicaban } & & \end{array}$

${ }^{30}$ El significado del término es una adaptación colonial de la acepción original. Hanks (2010: 130-132) presenta una serie de acepciones para esta palabra, entre ellas en contexto colonial se puede traducir como "convertir" o "creer". Se ha optado por el término "creer" debido a las implicaciones negativas que puede tener "convertir", que, según la Real Academia Española (2016), se define como "ganar a alguien para que profese una religión o la practique", lo que implica la influencia de un elemento externo que en época colonial son los frailes y los españoles y la eliminación del libre albedrío que no tiene lugar en "creencia" dado que pudieron tener la alternativa de creer o no creer. Es más probable el uso de este último término para dirigirse al Rey que el de "convertir".

31 Oc olal, según Hanks (2010: 132), se puede traducir por "creencia, tener fe" y en los diccionarios aparece como "fe" o "creencia” (Arzápalo, 1995: 1989; Barrera, 1980: 597). Debido a que este término y el anterior son iguales se ha optado por elegir "fe" evitando así repetir la palabra. 


\begin{tabular}{|c|c|c|}
\hline $\mathrm{ca}$ & ulzectahob & yalma ${ }^{11} /$ hthanil \\
\hline $\mathrm{ca}$ & u-bect-ah-ob & $y$-almah-than-il \\
\hline PART & 3sE-predicar-TRANS-COM-3pA & 3sE-decir-palabra-ABS \\
\hline
\end{tabular}

y nos predicaban el mandamiento de Dios.

Toon cahahalyamaobi,

toonca-hahal-yama-ob-i

1pP2 1pE-verdad-amar-3pA-CLIT

Nosotros los amamos de verdad, como si también fueran nuestros verdaderos

$x a[n]$ uai cahahal

xan ua-i ca-hahal

también como-CLIT 1pE-verdad

$\begin{array}{lllll}\text { yumobe, } & \text { baix } & \text { ucahob, } & \text { toon } & \text { bai } \\ \text { yum-ob-e } & \text { bay-ix } & \text { u-cah-ob } & \text { toon } & \text { bay } \\ \text { padre-PL-CLIT } & \text { así-CONJ } & \text { 3pE-hacer-3pE } & 1 \mathrm{pP} & \text { así }\end{array}$

padres, y así se nos hace por ellos, así nos

$\begin{array}{llll}\text { uyamailo } 12 / \text { nob } & \text { uhahal } & \text { mehene. } & \text { Tumenelob } \\ \text { u-yamail-on-ob } & \text { u-hahal } & \text { mehen-e } & \text { ti-u-men-el-ob } \\ \text { 3pE-amar-2pA-3pE3 } & \text { 3sE-verdad } & \text { hijo-CLIT } & \text { PREP-3pE-causa-3pE } \\ \text { aman como a verdaderos hijos. } & & \end{array}$

$\begin{array}{llll}\text { tun } & \text { kohol } & \text { yetel } & \text { ch/hlapahal } \\ \text { tan } & \text { u-kohol } & \text { y-etel } & \text { chap-ah-al- } \varnothing \\ \text { DUR } & \text { 3sE-enfermar-INC } & \text { 3sE-con } & \text { enfermar-INCO-INC-3sA }\end{array}$

Porque están enfermando e indisponiéndose ${ }^{32}$

$\begin{array}{llll}\text { ye[tel] } & \text { nupintabal } & \text { tume[n] } & \text { ciçin. } \\ \text { y-etel } & \text { nupin-t-ab-al } & \text { ti-u-men } & \text { ciçin } \\ \text { 3sE-con } & \text { contrariar-TRANS-PAS-INC-3sA } & \text { PREP-3sE-causa } & \text { demonio }\end{array}$

y son contrariados por causa del demonio,

$\begin{array}{ll}\text { 13/uppatcoonob } & \text { hach } \\ \text { u-ppat-c-oon-ob } & \text { hach } \\ \text { 3pE-dejar-INC-1pA-3pE } & \text { mucho } \\ \text { nos dejan muchos } & \end{array}$

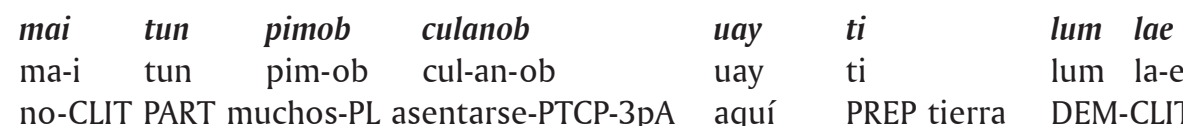
y no muchos están asentados aquí en esta tierra

${ }^{32} \mathrm{Al}$ igual que en la nota anterior, los términos kohol (Barrera, 1980: 411) y chaphal (Arzápalo, 1995, III: 1677; Barrera, 1980: 126) significan "enfermar". Se ha utilizado el sinónimo "indisponer" para evitar la repetición de la palabra. 


$\begin{array}{lllll}\text { yet }[\boldsymbol{e} / \boldsymbol{l i} & \text { xan } & \text { mata[n] } & \text { yulelob } & \text { uchaya[n] } \\ \text { y-etel-i } & \text { xan } & \text { matan } & \text { y-ul-el-ob } & \text { u-chayan } \\ \text { 3sE-con-CLIT } & \text { también } & \text { no } & \text { 3pE-llegar-INC-3pA } & \text { 3pE-los demás }\end{array}$

(y) tampoco llegan los demás

$\begin{array}{llll}\boldsymbol{t a} \boldsymbol{a}^{14} / \mathbf{l o b} & \boldsymbol{t e} & \text { tulumil } & \text { Castillae } \\ \text { tal-ob } & \text { te } & \text { ti-u-lum-il } & \text { Castilla-e } \\ \text { venir-3pE } & \text { PREP } & \text { PREP-3sE-tierra-ABS } & \text { Castilla-CLIT }\end{array}$

(que) vinieron de la tierra de Castilla

$\begin{array}{llll}\text { yokal } & \text { nachil } & \text { yetel } & \text { pikanil. } \\ \text { y-oklal } & \text { nach-il } & \text { y-etel } & \text { pik-an-il } \\ \text { 3sE-Rel } & \text { lejos-ADJ } & \text { 3sE-con } & \text { apartar-PTCP-ADJ }\end{array}$

porque está lejana y apartada.

\begin{tabular}{llllll} 
tayic & okotba & cacah & tech, & cech & Ahtepale, \\
tayic & okotba $^{33}$ & ca-cah & tech & c-ech & ah-tepal-e \\
razón & suplicar & $1 \mathrm{pE}-I N C d e c$ & $2 \mathrm{sP}$ & PRON-2sA & AG-rey-CLIT \\
\multicolumn{2}{l}{ Por esta razón te suplicamos a tí, tú que eres el Rey, } &
\end{tabular}

$\begin{array}{lll}\text { 15/ca } & \text { auokeçaich } & \text { tacpixan } \\ \text { ca } & \text { au-ok-eç-a[h]-ich- } \emptyset^{34} & \text { ti-ca-pixan } \\ \text { PART } & \text { 2sE-llorar-CAUS-COMdec-ojo-3sA } & \text { PREP-1pE-alma } \\ \text { que te compadezcas de nuestras almas } & \end{array}$

ca atu/m]te ca

ca a-tumut-e- $\varnothing$ ca a-tux-chi-t-e- $\varnothing$

PART 2sE-considerar-SUB-3sA PART 2sE-enviar-TRANS-SUB-3sA

(y) que consideres enviar

$\begin{array}{lllll}\text { frailes } & \text { fran/cis/cos } & \text { toon } & \text { payic } & \text { cabeel } \\ \text { frailes } & \text { franciscos } & \text { toon } & \text { pay-ic- } \varnothing & \text { ca-beel } \\ \text { frailes } & \text { franciscos } & 1 \mathrm{pP} & \text { guiar-SUB-3sA } & 1 \mathrm{pE}-\text { camino }\end{array}$

frailes franciscos a nosotros (que) guíen nuestro camino

33 El verbo ok generalmente se traduce por "llorar" (Arzápalo, 1995, III: 1997; Barrera, 1980: 601). Sin embargo, las adaptaciones coloniales modificaron el sentido del término, como indica Hanks (2010: 195), en “suplicar”.

${ }^{34}$ La acepción de $o k$ se modifica una vez más (Nota 32) por medio de ich que forma un nuevo término que significa "tener piedad" (Hanks, 2010: 195). En el documento en castellano aparece la palabra "compadezca" (Anexo 2, castellano, línea 12) que presenta el mismo sentido, por lo que se ha optado por la palabra utilizada en tal documento. 


$\begin{array}{llll}\text { yetel } & \text { cambeçicoon } & 16 / \text { tubelil } & \text { Dios, } \\ \text { y-etel } & \text { camb-eç-ic-oon } & \text { ti-u-bel-il } & \text { Dios } \\ \text { 3sE-con } & \text { enseñar-CAUS-INC-1pA } & \text { PREP-3sE-camino-Rel } & \text { Dios } \\ \text { y nos enseñen el camino de Dios, } & & \end{array}$

$\begin{array}{lcclcr}\text { unohlailobi } & \text { hi-mac } & \text { tiob } \text { yaln/hiob } & \text { uay } & \text { ti luum, } \\ \text { u-noh-lail-ob-i } & \text { hi-mac tiob yan-hi-ob } & \text { uay } & \text { ti luum } \\ \text { 3sE-especialmente-PL-CLIT IND-quien 3pP estar-COMdec-3pA } & \text { aquí } & \text { PREP tierra } \\ \text { especialmente quienes de ellos estuvieron aquí en la tierra, } & & \end{array}$

$\begin{array}{llllll}\text { binob } & \text { tuluumil } & \text { Castilla } & \text { tucate[n] } & \text { te } & \text { lae. } \\ \text { bin-ob } & \text { ti-u-luum-il } & \text { Castilla } & \text { ti-u-ca-ten } & \text { te } & \text { la-e } \\ \text { ir-3pA } & \text { PREP-3sE-tierra-CLIT Castilla } & \text { PREP-3sE-dos-vez } & \text { PREP } & \text { DEM-CLIT } \\ \text { y se fueron a la tierra de Castilla otra vez allí. } & \end{array}$

$\begin{array}{llll}{ }^{17} / \text { Laobi } & \text { hahal } & \text { ohelmail } & \text { cathaln], } \\ \text { laob-i } & \text { hahal } & \text { ohel-ma- } \varnothing \text {-il } & \text { ca-than } \\ \text { DEM-CLIT } & \text { verdad } & \text { saber-PERF-3sE-ABS } & \text { 1pE-lengua }\end{array}$

Ellos habían sabido verdaderamente nuestra lengua,

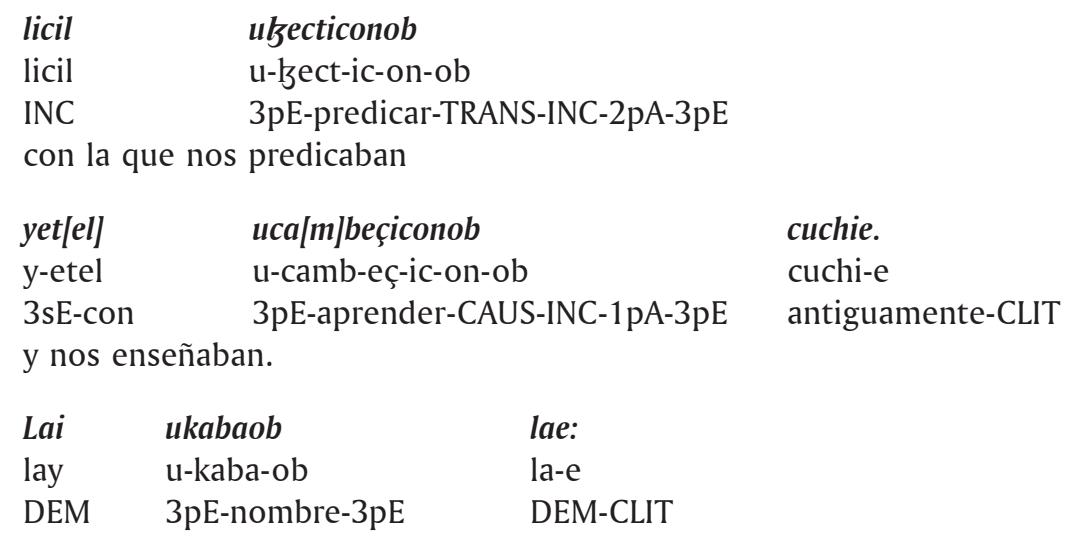

Estos son sus nombres:

18/fray Diego de Landa, fray Pedro Gumiel, tuprovinçiail Toledo, fray Diego de Landa fray Pedro Gumiel ti-u-provinçia-il Toledo fray Diego de Landa fray Pedro Gumiel PREP-3sE-provincia-ABS Toledo fray Diego de Landa, fray Pedro Gumiel, de la provincia de Toledo,

$\begin{array}{llll}\text { uhach } & \text { nohlaili } & \text { fray } & \text { Diego de La/n]dae, } \\ \text { u-hach } & \text { noh-lail-i } & \text { fray } & \text { Diego de Landa-e } \\ \text { 3sE-muy } & \text { especialmente-CLIT } & \text { fray } & \text { Diego de Landa-CLIT }\end{array}$

muy especialmente este fray Diego de Landa, 
19/tume[n] unohol uyabal utibilil yet[el] yubil, ti-u-men u-nohol u-yabal u-tibil-il y-etel y-ub-il REP-3sE-Rel 3sE-gran 3sE-mucho 3sE-bondad-ABS 3sE-con 3sE-bueno-ABS por la grandeza (y) la abundancia de sus virtudes y bondad,

$\begin{array}{llll}\text { tuuich } & \text { cayumil } & \boldsymbol{t i} & \text { Dios } \\ \text { ti-u-uich } & \text { ca-yum-il } & \text { ti } & \text { Dios } \\ \text { PREP-3sE-ojo } & \text { 1pE-padre-Rel } & \text { 3sP } & \text { Dios }\end{array}$

a los ojos de nuestro padre Dios.

lai. Hach yaabil capay ti tuhunal tac ${ }^{20} /$ chr[ist]ianoile. la-i hach yaab-il ca-pay ti t-u-hunal ti-ca-cristiano-il-e DEM-CLIT muy mucho-CLIT 1pE-deuda 3sP PREP-3sE-sólo PREP-1pE-cristiano-ABS-CLIT

Muy abundante (es) nuestra deuda con él solamente por nuestro cristianismo.

$\begin{array}{llllll}\text { Yetel } & \text { Fray } & \text { Miguel de la Puebla } & \text { yetel } & \text { uchayan } & \text { padresob } \\ \text { y-etel } & \text { fray } & \text { Miguel de la Puebla } & \text { y-etel } & \text { u-chayan } & \text { padres-ob } \\ \text { 3sE-con } & \text { fray } & \text { Miguel de la Puebla } & \text { 3sE-con } & \text { 3sE-demás } & \text { padres-PL }\end{array}$

Y Fray Miguel de la Puebla y los demás padres

$\begin{array}{lllc}\text { bahun } & \text { auolah, } & \text { cech } & \text { Ahtepa }^{21} / \text { le. } \\ \text { bahun } & \text { au-ol-ah- } \varnothing & \text { c-ech } & \text { ah-tepal-e } \\ \text { cuantos } & \text { 2sE-querer-INCdec-3sA } & \text { PRON-2sA } & \text { AG-rey-CLIT }\end{array}$

cuantos quieras, tú que eres el Rey.

$\begin{array}{lll}\text { Yoklalix } & \text { canaat } & \text { mail } \\ \text { yoklal-ix } & \text { ca-naat } & \text { ma-il } \\ \text { porque-CONJ } & 1 \mathrm{pE}-\mathrm{pntender}^{-} & \text {no-ABS }\end{array}$

Y porque entendemos

$\begin{array}{lllll}\text { yet[el] } & \text { ocanil } & \boldsymbol{t i} & \text { col } & \text { mail } \\ \text { y-etel } & \text { oc-an-il } & \text { ti } & \text { ca-ol } & \text { ma-il } \\ \text { 3sE-con } & \text { entrar-PTCP-ABS } & \text { PREP } & 1 \mathrm{pE}-\text { corazón } & \text { no-ABS } \\ \text { y creemos } & & & & \end{array}$

35 La construcción ocanil ti col está formada por dos palabras diferentes: $o l$, que se define como “corazón”, refiriéndose al corazón formal y no al material (Arzápalo, 1995, III: 1999; Barrera, 1980: 603-604), y el verbo oc que significa "entrar" (Arzápalo, 1995, III: 1989). Su traducción literal sería “entrar en el corazón”, pero los cambios en los significados de las palabras en época colonial dieron lugar a "creer" (Hanks, 2010: 193). 
$\begin{array}{lll}\text { çamac } & \text { pakmabac } & \text { talnllah } \\ \text { çamac } & \text { pakmab-ac- } \varnothing & \text { tanlah } \\ \text { tiempo } & \text { vano-SUB-3sA } & \text { servicio }\end{array}$

que en algún tiempo no sería en vano el servicio

lic culzcinic tech, cech 22/Ahtepale.

lic ca-ub-cin-ic- $\varnothing$ tech c-ech ah-tepal-e

INC 1pE-hacer algo bien-CAUS-INC-3sA 2sP PRON-2sA AG-rey-CLIT

que te hacemos bien a tí, tú que eres el Rey,

$\begin{array}{llll}\text { uloltoon } & \text { tachrlist]ianoil } & \text { pucçikal } & \text { tulacal. } \\ \text { ul-ol-t-oon } & \text { ti-ca-cristiano-il } & \text { pucçikal } & \text { t-u-lacal } \\ \text { querer-TRANS-2pA } & \text { PREP-2sE-cristiano-ADJ } & \text { corazón } & \text { PREP-3sE-todo }\end{array}$

que nos quieres por tu corazón cristiano a todos.

Baix alanil col binil antabacoon

bay-ix al-an- $\varnothing$-il ca-ol bin-il anat-ab-ac-oon

así-CONJ decir-PTCP-3sA-ABS 2pE-corazón FUT-INC favorecer-PAS-SUBdec-1pA

Y así confiamos que seremos favorecidos

tamen 23 tuçebal, cech Ahtepale.

ti-a-men ti-u-çeb-al c-ech ah-tepal-e

PREP-2sE-ABL PREP-3sA-presto-ABS PRON-2sA AG-rey-CLIT

por ti con presteza, tú que eres el Rey.

Cayumil ti Dios çaçcunic yetel bailcunic

ca-yum-il ti Dios çaç-cun-ic- $\emptyset$ y-etel bail-cun-ic- $\varnothing$

1pE-padre-ABS 3sP Dios iluminar-CAUS-COM-3sA 3sE-con perseverar-CAUS-COM-3sA

Que nuestro padre Dios ilumine y persevere

unachcu ${ }^{24} /$ nic auik tutanlahil.

u-nach-cun-ic- $\varnothing$ au-ik ti-u-tan-lah-il

3sE-extender-CAUS-COM-3sA 2sE-espíritu PREP-3sE-servir-INCO-SUST

extendiendo tu espíritu ${ }^{36}$ en su servicio.

\begin{tabular}{|c|c|c|c|c|}
\hline Yucatan, & tuhoppel & yuil & março & 1567 \\
\hline Yucatán & ti-u-ho-ppel & $y-u-i l$ & março & 1567 \\
\hline Yucatán & PREP-3sE-cinco-CLAS & 3sE-mes-Rel & marzo & 1567 \\
\hline
\end{tabular}

${ }^{36}$ La construcción en castellano es "y aumente siempre en su servicio" (Anexo 2, castellano, línea 18) y como se puede ver no existen los elementos necesarios en el texto para traducir esta expresión. La adaptación de una expresión habitual en los documentos castellanos es una afirmación más de la existencia de un modelo sobre el que se basaron para la realización del documento. 
${ }^{25} / \mathrm{C}[$ atólica] R[eal] Mag[estad]

"Católica Real Majestad"

26/uchinamilob

achinam,

u-chinam-il-ob

a-chinam ${ }^{37}$

3pE-vasallo-REL-3pE

2sE-pueblo

"Los vasallos de tus pueblos, tus sirvientes,

$27 /$ usbenic

uoben-ic- $\emptyset^{38}$

acilich

kabob,

a-cilich kab-ob

olfatear-COM-3sA

2sE-santa

mano-PL

besan tus santas manos, tú que eres el Rey." auahtanlahulob,

au-ah-tanlah-ul-ob

2sE-AG-servir-SUST-PL

Fol. 1v.

$1 /$ (cruz)

2/ Católica Real Majestad

3/ Porque todos los vasallos de Vuestra Majestad, entendemos el deseo que es que todos nos salvemos tiene, y para esto

4/ provee siempre Vuestra Majestad en sus reinos de ministros suficientes, para que alumbren y enseñen a los que no saben.

5/ Y aunque nosotros estamos apartados de esos reinos, entendemos, mas tiene Vuestra Majestad, el mismo cuidado que si

\% estuviésemos cercanos. Y que huelga de ser avisado de lo que más nos convenga conforme a nuestra bajeza de

7/ ingenio y pobreza de bienes temporales. Por tanto, hacemos saber a Vuestra Majestad, que desde el principio de nuestra

8/ conversión a la fe, hemos sido doctrinados y enseñados de frailes franciscos y ellos con su doctrina y pobreza,

$9 /$ nos han predicado y predican la ley de Dios. Y los amamos como a verdaderos padres y ellos a nosotros nos tienen

${ }^{37}$ La fórmula castellana "humildes vasallos y siervos" (Anexo 2, castellano, línea 21) se ha adaptado al maya empleando el término chinam que a lo largo del texto se ha traducido por "vasallos" (Nota 19), aunque en este caso se ha incluido su segunda acepción "pueblos" para adecuar la expresión a la lengua maya.

${ }^{38}$ El verbo uəben (Arzápalo, 1995, III: 2149; Barrera, 1980: 902) significa "oler" o "besar oliendo como hacen los indios y el tal beso". Aunque se ha traducido el término como "besar", ya que la fórmula castellana es "que sus reales manos besan" (Anexo 2, castellano, línea 22); existe la posibilidad de que la acción de besar la mano como gesto de respeto se haya confundido con la acción de oler u olfatear y de ahí la utilización de este verbo. Enlazando tal idea (comunicación personal, Alfonso Lacadena, marzo de 2015) se deber tener en cuenta cuál era el tipo de saludo o tratamiento afectivo habitual entre los indígenas. Tomando como ejemplo a los inuit y otras culturas americanas que usaban el frotamiento de nariz, similar a la acepción que aparece en el diccionario, podría darse un sentido diferente a este verbo y a las implicaciones sociales a las que se refiere. 
10/ como a hijos. Y con enfermedades y persecuciones del demonio y sus secuaces, han quedado muy pocos. Y

11/ también por no venir de España a esta tierra como cosa apartada, por esta causa,

12/ se compadezca de nuestras ánimas y nos envíe frailes franciscos que nos guíen y enseñen la carrera de Dios y

13/ en especial a algunos que de estas partes han ido a España, que sabían muy bien nuestra lengua en la que nos predica-

14/ ban. Que se llaman: fray Diego de Landa, fray Pedro Gumiel de la provincia de Toledo y en especial fray Diego de

15/ Landa, que por su mucha santidad y bondad del pueblo de Dios, debemos a él sólo toda nuestra cristiandad. Y fray Mi-

16/ guel de la Puebla y los demás que Vuestra Majestad mandare. Y porque entendemos, hacemos en esto servicio a Vuestra Majestad

17/ que con tan cristiano corazón, nos desea todo bien. Quedamos confiados seremos con brevedad favorecidos

18/ de Vuestra Majestad, a quien nuestro Señor alumbre y aumente siempre en su servicio. De Yucatán y de marzo, cinco

19/ de 1567 años

20/ Católica Real Majestad

21/ Humildes vasallos y siervos de Vuestra Majestad

22 / que sus reales manos besan. 